UNIVERSIDADE DE SÃO PAULO

FACULDADE DE FILOSOFIA CIÊNCIAS E LETRAS DE RIBEIRÃO PRETO PROGRAMA DE PÓS-GRADUAÇÃO EM FÍSICA APLICADA À MEDICINA E BIOLOGIA

\title{
Avaliação da qualidade da imagem e taxa de exposição na cardiologia intervencionista
}

Roberto Contreras Pitorri

Dissertação apresentada à Faculdade de Filosofia, Ciências e Letras de Ribeirão Preto da Universidade de São Paulo, como parte das exigências para a obtenção do título de Mestre em Ciências.

Ribeirão Preto -SP 


\section{ROBERTO CONTRERAS PITORRI}

\section{Avaliação da qualidade da imagem e \\ taxa de exposição na \\ cardiologia intervencionista}

Dissertação apresentada à Faculdade de Filosofia, Ciências e Letras de Ribeirão Preto da Universidade de São Paulo, como parte das exigências para a obtenção do título de Mestre em Ciências.

\section{Área de Concentração:}

Física Aplicada à Medicina e Biologia.

\section{Orientador:}

Adelaide de Almeida

\section{Versão Original}

Disponível na FFCLRP-USP

Ribeirão Preto - SP 
AUTORIZO A REPRODUÇÃO E DIVULGAÇÃO TOTAL OU PARCIAL DESTE

TRABALHO, POR QUALQUER MEIO CONVENCIONAL OU ELETRÔNICO, PARA FINS DE ESTUDO E PESQUISA, DESDE QUE CITADA A FONTE.

Pitorri, Roberto C

Avaliação da qualidade da imagem e taxa de exposição na

Cardiologia intervencionista/Roberto C Pitorri; orientadora

Adelaide de Almeida. Ribeirão Preto, 2013. 47 p.

Dissertação de Mestrado - Programa de Pós-Graduação em Física Aplicada à Medicina e Biologia - Faculdade de Filosofia Ciências e Letras de Ribeirão Preto da Universidade de São Paulo.

Inclui Bibliografia

Palavras chave: fluoroscopia, qualidade da imagem, taxa de exposição, otimização 
Roberto C Pitorri

Título: Avaliação da qualidade da imagem e taxa de exposição na cardiologia intervencionista.

Dissertação apresentada à Faculdade de Filosofia, Ciências e Letras de Ribeirão Preto da Universidade de São Paulo, como parte das exigências para a obtenção do título de Mestre em Ciências.

Aprovado em: I

\section{Banca Examinadora}

Prof. Dr. : Instituição:

Julgamento: Assinatura:

Prof. Dr. : Instituição:

Julgamento: Assinatura:

Prof. Dr. Instituição:

Julgamento: Assinatura: 


\section{Dedicatória}

Dedico este trabalho à minha família, em especial aos meus filhos. 


\section{Agradecimentos}

Agradeço à minha família, principalmente aos meus filhos, pela compreensão em tantos momentos delicados.

Aos meus professores, em especial à minha orientadora, Profa Adelaide, pelos seus comentários de estímulo e de apoio .

Aos meus amigos pela presença, mesmo ausentes.

Aos que acreditaram nos meus esforços por realizar um bom trabalho, desde os tempos do Sr Aristides Negretti, ao Prof Dr Sérgio Mies, ao Pedro Stern (Siemens), e atualmente aos clientes da Optimagem que financiaram este projeto.

À Universidade de São Paulo, Faculdade de Filosofia Ciências e Letras de Ribeirão Preto, FAMB, pela oportunidade e estrutura oferecidas. 


\section{Resumo}

Pitorri, R. C. Avaliação da qualidade da imagem e taxa de exposição na cardiologia intervencionista. 47p. Dissertação - Faculdade de Filosofia Ciências e Letras de Ribeirão Preto, Universidade de São Paulo, Ribeirão Preto, 2013

A Fluoroscopia é uma técnica de obtenção de imagens de raios $\mathrm{X}$, através de um detector de imagens dinâmicas, que permite o acompanhamento de exames de órgãos em tempo real. Os detectores atualmente utilizados são os intensificadores de imagem (II) e os Flat Panel (FP), os primeiros (do tipo válvula) têm a principal função de aumentar o brilho na imagem e mais recentemente os segundos (de estado sólido), também sido utilizados nos equipamentos de fluoroscopia para melhorar a qualidade da imagem (contraste e detalhe), diminuindo ruídos e artefatos na mesma. Os exames gerais que utilizam a técnica de fluoroscopia servem para cabeça, tórax e abdômen, que antes eram realizados com um mesmo tipo de equipamento, mas devido à evolução da tecnologia equipamentos dedicados a esses exames passaram a ser utilizados. O objetivo deste trabalho é analisar especificamente um grupo de equipamentos de fluoroscopia cardíaca (de diferentes instituições e fabricantes) em número de 19 equipamentos, sendo 10 que utilizam detector II e 9 que utilizam detector FP, para inferir como estão os parâmetros de contraste, detalhe e taxa de exposição no detector (também interessante para os serviços de manutenção) em relação a sua média e também como estão em relação às referências internacionais. Para tal, foi desenvolvido um objeto simulador e um protocolo de testes incluindo medidas de taxa de exposição e análises dos parâmetros obtidos, a saber: a) testes preliminares para aceitação do equipamento para a amostragem, b) de detalhe e de contraste (utilizando o objeto simulador desenvolvido) para a obtenção do seu produto denominado por FOM (Figure of Merit), c) as medidas de taxa de exposição que chega no detector e d) as análises das distribuições dos resultados obtidos com os dois grupos de detectores, quanto as suas médias e comparação dessas (equipamentos utilizados no Brasil) com os valores de referência da literatura internacional. Do trabalho realizado foi possível comprovar que o objeto simulador e o protocolo desenvolvido, juntos à metodologia aplicada, foram 
adequados para auxiliar no controle de qualidade dos equipamentos selecionados, classificando-os quanto aos potenciais de otimização de FOM e à taxa de exposição na entrada do detector de imagem, TEEDI. Os FOMs médios do II e do FP distam do FOM referência de 35,5\% e 35,0 \% e as TEEDIs médias para os II e FP distam da TEDDI referência respectivamente de $13,8 \%$ e $24,9 \%$. Estes últimos deverão ser ajustados pela manutenção para trazê-los mais próximos das referências utilizadas nas distribuições obtidas.

Palavras chave: fluoroscopia, qualidade da imagem, taxa de exposição, otimização 


\section{Abstract}

Pitorri, R.C.. Evaluation of Image Quality and exposure rate in interventional cardiology, 47 p. Dissertation (MSc) - Faculdade de Filosofia, Ciências e Letras de Ribeirão Preto, Universidade de São Paulo, Ribeirão Preto, 2013

Fluoroscopy is a $\mathrm{X}$ ray technique used to obtain images through a dynamic image detector or sensor that allows to follow the organs movements in real time exams. Nowadays the detectors used are the image intensifier (II) e the Flat Panel (FP). The first one type valve has the main function to enhance the image brightness and more recently was developed the second one, (solid state technology) which the main function is to enhance the image quality (contrast and detail) minimizing the noise and artifacts in itself. Head, thorax and abdomen are the body sections which the general fluoroscopy deals and that was performed with one only type of equipment. Actually these exams are performed with dedicated machines due to the technology evolution and several manufactures are responsible for theirs development and assembly in several continents (Americas, Europe and Asia). The scope of this work is to analize two groups of fluoroscopy equipments (II and FP detectors), dedicated only for cardiac fluoroscopy and from different institutions and manufacturers, in order to infer how the parameters of contrast, detail and exposure rate at the entrance of the detector are in a Quality Control that the maintenance service would be also interested, besides a medical physicist. With these results one could know how the cited groups (through their average results) would be doing related to others groups of equipments or an specific one and even to international references. With this purpose a PMMA simulator object (OS) was developed with an protocol derived from the literature that was composed of exposure rate in the entrance of the detector (TEEDI), tests related to the selection of the equipments to be part of theirs samples, tests of contrast and detail (using the OS) to obtain their product named by FOM (Figure of Merit) and with all results obtained, to analyse the two distribution groups through their averages and comparing them not only with themselves, but also with the references from abroad. With this work it was possible to confirm that the OS, as well the protocol developed together the methodology used, were adequate to perform the quality control of the selected 
equipment samples, classifying them related theirs optimization potentials of FOM and TEEDI. The average FOMs for II and FP are far from the reference by 35,5\% and 35,0 \% respectively and the average TEEDIs for II and FP are far from the reference TEDDI respectively by $13,8 \%$ and $24,9 \%$. These last one has to be adjusted for the maintenance service (mainly the FP one) in order to bring them more near to the reference used to obtain the distributions.

Keywords: fluoroscopy, image quality, exposure rate, optimization 


\section{Lista de Figuras}

\section{Capítulo 2:}

1. O inventor Thomas Alva Edison em seu laboratório, trabalhando no desenvolvimento do

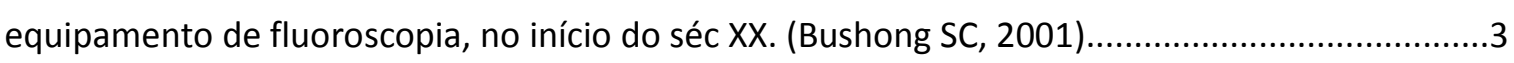

2. Equipamento precursor da fluoroscopia com écran .........................................................

3. Exemplo de imagens Fluoroscópicas clínicas do coração. Na esquerda há maior borrosidade cinética na imagem e na direita a redução do tempo de exposição melhorou a sua qualidade......6

4. Equipamento atual de fluoroscopia com o arco em C, o detector FP, os monitores, o tubo de raios $\mathrm{X}$ e a mesa de procedimentos

5. O detector FP e suas partes principais.

6. Detector flat panel utilizado em fluoroscopia com a unidade de controle lógico, a unidade de processamento e apresentação da imagem e a unidade de arquivo.

7. Imagem RAW de detector Flat Panel apresentando os pontos sem sinal ou dead pixels......

8. Paciente apresentando eritema radio induzido pós procedimento de cardiologia intervencionista, onde a área afetada no braço direito é exemplo de dose evitável.. 15

9. Imagem do objeto simulador CD DENT para avaliar os parâmetros limiar de contraste e limiar de detalhe.

\section{Capítulo 3:}

1. Fotografia do dispositivo utilizado para medir a quilovoltagem pico dos equipamentos. .20

2 a) vista superior do Objeto Simulador desenvolvido e b) foto digital da visão lateral do Objeto Simulador...... 23

\section{Capítulo 4:}

1. Detalhe II $\mathrm{X}$ Identificação do Equipamento, com seus respectivos códigos e o valor médio dos valores encontrados na distribuição

2. Detalhe FP x Identificação do Equipamento com seus respectivos códigos e o valor médio dos valores encontrados na distribuição

3. Contraste II $x$ Equipamento com seus respectivos códigos e o valor médio dos valores encontrados na distribuição. 
4. Contraste FP $x$ Equipamento com seus respectivos códigos e o valor médio dos valores encontrados na distribuição

5. FOM II x Identificação do Equipamento com seus respectivos códigos e o valor médio dos valores encontrados na distribuição .30

6. FOM FP x Identificação do Equipamento com seus respectivos códigos e o valor médio dos valores encontrados na distribuição 31

7. TEEDI II x Identificação do Equipamento com seus respectivos códigos e o valor médio dos valores encontrados na distribuição. .32

8. TEEDI FP x Identificação do Equipamento com seus respectivos códigos e o valor médio dos valores encontrados na distribuição.

9. Resultados das medições de FOM em função das medições de TEEDI para o detector II. Os pontos representam os valores medidos para cada equipamento e as retas horizontais são os valores médios e de referencia para o FOM. As retas verticais são respectivamente os valores médio e de referência para a TEEDI. 34

10. Resultados das medições de FOM em função das medições de TEEDI para o detector FP. Os pontos representam os valores medidos para cada equipamento e as retas horizontais são os valores médios e de referência para o FOM. As retas verticais são respectivamente os valores médio e de referência para a TEEDI. 


\section{Lista de Abreviaturas}

AAPM American Association of Physicists in Medicine

ABC Automatic Brightness Control

AEC Automatic Exposure Control

ALARA At Least As Reasonable Achievable

CD Contraste Detalhe

CI Câmara de Ionização

CNEN Comissão Nacional de Energia Nuclear

FOM Figure of Merit

FP Flat Panel

ICRP International Commission of Radiation Protection

II Intensificador de Imagem

IPEN Instituto de Pesquisas Energéticas e Nucleares

OS Objeto Simulador

DDP Diferença de Potencial 


\section{Sumário}

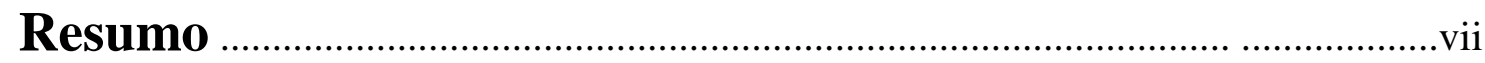

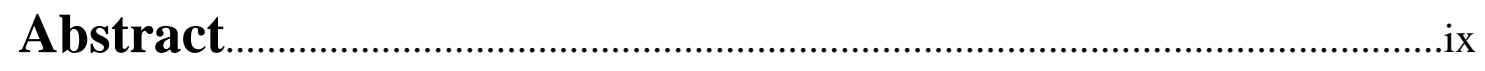

Capítulo 1

Introdução..

\section{Capítulo 2}

Noções Teóricas

$$
\begin{aligned}
& \text { Breve histórico da Fluoroscopia } \\
& \text { Intensificador de Imagens } \\
& \text { Detectores Flat Panel } \\
& \text { Aplicações da fluoroscopia } \\
& \text { Cardiologia Intervencionista } \\
& \text { Estado de otimização } \\
& \text { Avaliação da qualidade }
\end{aligned}
$$

\section{Capítulo 3}

Materiais e Métodos.

\section{Materiais}

Métodos

\section{Capítulo 4}

Resultados e Discussões

$$
\begin{aligned}
& \text { Resultados de Detalhe x Equipamento } \\
& \text { Resultados de Contraste x Equipamento } \\
& \text { Resultados de FOM x Equipamento } \\
& \text { Resultados de TEEDI x Equipamento } \\
& \text { Resultados de FOM } x \text { TEEDI } \\
& \text { Valores de referência }
\end{aligned}
$$

\section{Capítulo 5}

Conclusões. 


\section{Capítulo 1}

\section{Introdução}

A medicina foi beneficiada com a descoberta dos raios X por Roentgen em 1895 e a fluoroscopia por Thomas Alva Edson que tem sido utilizada em número crescente de exames desde então. As primeiras aplicações da fluoroscopia na medicina, foram aquelas cujo objetivo era o de diagnosticar lesões em estágios iniciais de forma a maximizar as chances de sucesso do tratamento. Os exames foram se relacionando a alguns tratamentos e obtiveram sucessos ao reduzir os riscos de intervenções cirúrgicas do coração (pontes de safena, valvoplastia etc...), sem a necessidade de anestesia geral. Dada a elevada complexidade do procedimento da intervenção, a exposição também é elevada e há relatos de um número importante de efeitos biológicos em pacientes e equipe envolvida (Miller et al, 2012; ICRP, 2013) , fazendo com que a comunidade de físicos em medicina voltasse sua atenção ao problema, na tentativa de reduzir as exposições aos pacientes e assim reduzir os riscos de efeitos biológicos, embora enfrentando o desafio de manter a qualidade da imagem necessária aos serviços médicos ao paciente. As taxas de exposição a serem utilizadas e que chegam nos detectores fornecem proporcionalmente doses absorvidas ao paciente, mas devem ser as mínimas possíveis que ainda possam proporcionar a qualidade necessária e suficiente para aumentar as chances de se fazer diagnósticos de lesões sutis e do sucesso do tratamento com o mínimo possível de exposição ao paciente e equipe. $\mathrm{O}$ desafio ao sugerir a redução na taxa de exposição no detector é: além de atender ao princípio ALARA (doses tão baixas quanto razoavelmente exequível) (ICRP, 1991) também manter a qualidade da imagem (Lin et al, 2012). Portanto, qualquer tentativa de otimização deverá ter uma metodologia de controle da qualidade para garantir que o processo está sendo optimizado e que há uma melhora nos resultados (Simon et al, 2008). Neste trabalho o objetivo é propor uma forma de avaliar quantitativamente o potencial de otimização dos equipamentos dedicados à cardiologia intervencionista, no desenvolvimento de um protocolo que contivesse os testes para aceitação inicial dos equipamentos de amostragem, os testes de qualidade da imagem (contraste e detalhe) e as 
medidas da taxa de exposição no detector de imagem (Anderson et al, 2000). Pode-se afirmar que, mantendo as condições de operação do equipamento inalteradas, uma redução da taxa de exposição no detector causaria a redução na dose absorvida ao paciente e também na dose absorvida à equipe (ICRP, 2013). Da análise envolvendo o contraste e o detalhe, através do seu produto representado pelo parâmetro FOM (Figure of Merit) e a taxa de exposição, também considerando as referências da literatura quanto ao FOM e taxa de exposição (Boone et al, 1993), foi possível avaliar semi-quantitativamente o desempenho dos equipamentos e de um equipamento escolhido aleatoriamente em relação ao grupo de equipamentos e em relação ao esperado internacionalmente (Cowen, 1987; Ishibashi , 2011; Miller, 2012) . Dos resultados obtidos pode-se inferir potenciais de otimização para ambos os grupos de detectores, intensificador de imagem II e Flat Panel FP, dos ajustes dos equipamentos que podem ser comunicados diretamente aos responsáveis pela manutenção. Este protocolo foi criado para o físico médico fazer a avaliação dos equipamentos de fluoroscopia cardíaca e serve também para treinamento de toda a equipe envolvida (técnicos, médicos radiologistas e serviço de manutenção) (ICRP, 2013) que munidos do resumo de todos os testes e medidas através das distribuições de FOM versus Taxa de Exposição na Entrada do Detector de Imagens (TEEDI), para os tipos de detectores de interesse, serão capazes de indicar quais os parâmetros necessários para serem ajustados nos equipamentos.

Este trabalho visa contribuir neste desafio, na revisão do ajuste da taxa de exposição no detector, se há algum potencial de otimização em termos de exposição ou de qualidade, analisando uma amostra de equipamentos e posicionando-os em relação a valores médios e de referência. Os fundamentos teóricos necessários para o desenvolvimento deste trabalho são apresentados no Capítulo 2 - Noções Teóricas e no Capítulo 3 - Materiais e Métodos. No Capítulo 4 estão presentes os Resultados e as discussões relacionadas ao Protocolo proposto e no Capítulo 5 tem-se as conclusões inferidas do trabalho. 


\section{Capítulo 2}

\section{Noções Teóricas}

\section{Breve histórico da fluoroscopia}

A geração de radiografias, na época da descoberta dos raios $\mathrm{X}$, era muito limitada pela tecnologia disponível naquele momento. Com o surgimento da fluoroscopia, a possibilidade de visualizar os órgãos do paciente em tempo real tornou-se possível e muitos avanços nesta técnica passaram a ser registrados em todo o mundo. A fluoroscopia é uma modalidade de geração de imagens médicas, a qual emprega, na sua origem, raios $\mathrm{X}$ de forma semelhante ao equipamento convencional de produção de radiografias. A diferença é que o detector de imagens estáticas foi substituído por um detector de imagens dinâmicas, o qual apresenta imagens em tempo real dos movimentos dos órgãos internos do paciente.

As pesquisas com fluoroscopia datam de 1900, quando o inventor Thomas Alva Edison e seu assistente, Figura 1, trabalharam intensamente na idéia de uma tecnologia que permitisse a observação dos movimentos dos órgãos do corpo humano.

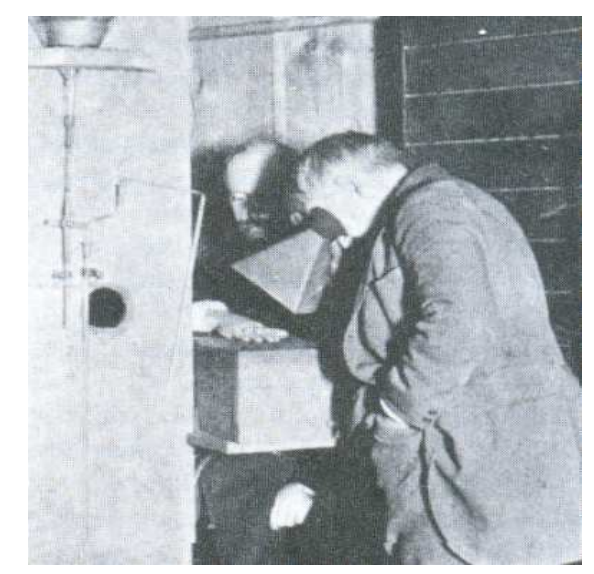

Figura 1. O inventor Thomas Alva Edison em seu laboratório, trabalhando no desenvolvimento do equipamento de fluoroscopia, no início do séc XX (Bushong, 2001). 
Em sua história, a modalidade foi marcada por muitos esforços de pesquisadores para melhorar a visibilidade das estruturas do paciente e a proteção radiológica da equipe. O uso do écran de cristal fluorescente só teve alternativa a partir dos anos 60, com o desenvolvimento da tecnologia do intensificador de imagens, que se trata, em resumo, de uma válvula com $25 \mathrm{~cm}$ de diâmetro e $40 \mathrm{~cm}$ de altura com vácuo em seu interior e lentes eletrônicas para focalização e aumento do brilho da imagem. Quando se utilizava o equipamento de fluoroscopia equipado com o écran fluorescente como detector onde a imagem era formada, o médico precisava antes adaptar sua visão ao escuro e para tanto, utilizava óculos escuros especiais e aguardava 15 minutos em média para iniciar o exame. Os procedimentos eram conduzidos em ambiente iluminado apenas por uma lâmpada vermelha de $15 \mathrm{w}$. Nestas condições a visão é limitada, pois o sistema visual humano, para aumentar a sua sensibilidade à luz, abre a pupila ao máximo e passa a usar a região periférica da retina, composta na maioria de bastonetes. Assim, o sistema visual humano passa a detectar imagens, mesmo em condições de baixas intensidades de luz, mas a visibilidade de detalhes é reduzida com este recurso e o uso da visão noturna em fluoroscopia com écran resultava em uma visão limitada de detalhes, já que a imagem era detectada pelos bastonetes na retina (Bromley, 1944). Atualmente os fabricantes de detectores usam um recurso parecido: o binning, que é o uso de mais de um pixel para detectar a luz, aumentando a área de detecção e também a sensibilidade do sistema. Na Figura 2 é apresentado um fluoroscópio convencional com écran.

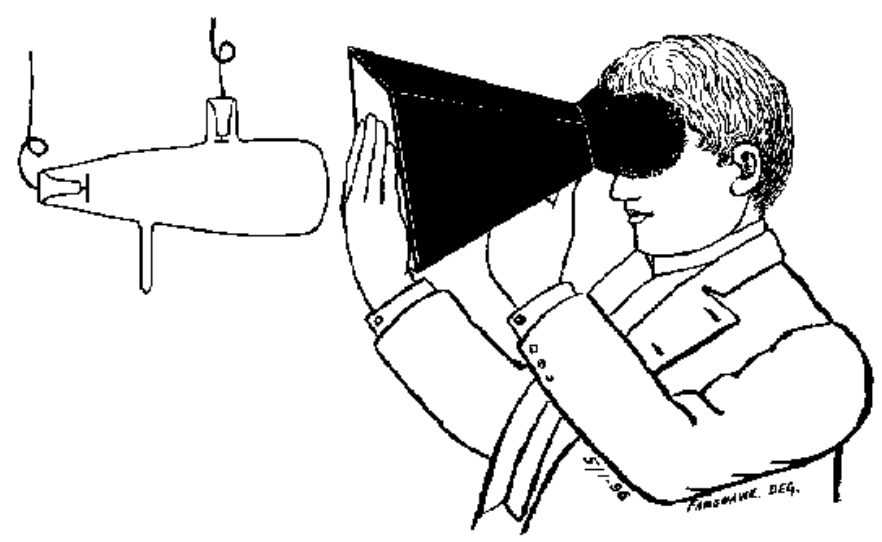

Figura 2. Equipamento precursor da fluoroscopia com écran (http://www.emory.edu/X-RAYS/century.htm acessado em 20 de agosto de 2013). 
Os pesquisadores precisavam resolver um problema complexo para a época: o ajuste automático dos parâmetros de exposição: a tensão aplicada ao tubo de raios $\mathrm{x}$, a corrente do tubo e o tempo de exposição, de forma que o brilho no écran fosse adequado a cada região do paciente que estivesse sendo examinada e ainda, que este brilho fosse constante, para o caso de ser necessário obter uma radiografia. Quando o médico examinava o tórax do paciente e precisava examinar o abdôme em seguida, movimentava o fluoroscópio e o aumento da atenuação fazia com que fosse necessário aumentar os parâmetros de exposição no gerador. Isto tomava muito tempo do procedimento porque, a cada mudança da posição do equipamento, um novo ajuste dos parâmetros era necessário. Esta dificuldade levava à solução mais imediata: ajustar os parâmetros de tensão e corrente para as regiões de maior atenuação e que resultasse em brilho adequado e deixá-los inalterados, para as regiões de menor atenuação, o que gerava brilho mais intenso, embora mesmo com a tecnologia atual isto ainda ocorre. É interessante salientar que, com esta prática, tanto o paciente quanto a equipe estavam expostos à radiação $\mathrm{X}$, com doses desnecessariamente elevadas e evitáveis (Balter, 2006; Lemoigne et al, 2009).

\section{Principais componentes do equipamento de fluoroscopia}

O equipamento de fluoroscopia deve possuir um gerador de radiação, com potência mínima de 0,6 kW para regime de emissão contínua, possibilitar diferenças de potêncial de 60 a $120 \mathrm{kV}$ e corrente de $5 \mathrm{~mA}$. A potência do gerador é uma característica que deve ser adequada aos diferentes tipos de aplicação, para as quais esta técnica será utilizada. O equipamento utilizado em traumatologia, como exemplo, pode ter potência menor em comparação com o de radiologia intervencionista, onde há a necessidade de maior potência. Mais especificamente, o equipamento para cardiologia deve ter potência entre 70 e $100 \mathrm{~kW}$. (Nickoloff e Strauss, 2001). A técnica de fluoroscopia pulsada, onde a corrente é aumentada por um intervalo de tempo da ordem de ms (milésimos de segundo), numa frequência programável pelo técnico, da ordem de 5 a 30 imagens por segundo (im/s) é requisito fundamental para esta aplicação (Canevaro, 2009). O estado da arte em geradores de radiação atualmente é a tecnologia de gerador de alta frequência (da ordem de 10 a 20 $\mathrm{kHz}$ ) que possibilita a programação da técnica de fluoroscopia pulsada, a qual é 
fundamental em cardiologia para reduzir a borrosidade cinética nas imagens, que tem origem nos movimentos cardíacos. A velocidade com que o músculo e as artérias coronárias se movem é da ordem de $10 \mathrm{~cm} / \mathrm{s}$, mas quando há a necessidade clínica de estudar as válvulas cardíacas, para o diagnóstico de insuficiência mitral e em procedimento intervencionista de valvoplastia (reconstrução de válvulas mitral e aórtica), as velocidades das cúspides valvares chegam a $100 \mathrm{~cm} / \mathrm{s}$ e o equipamento deve ter a possibilidade de emitir pulsos de radiação curtos, da ordem de 1ms. A Figura 3 mostra exemplos de imagens clínicas.
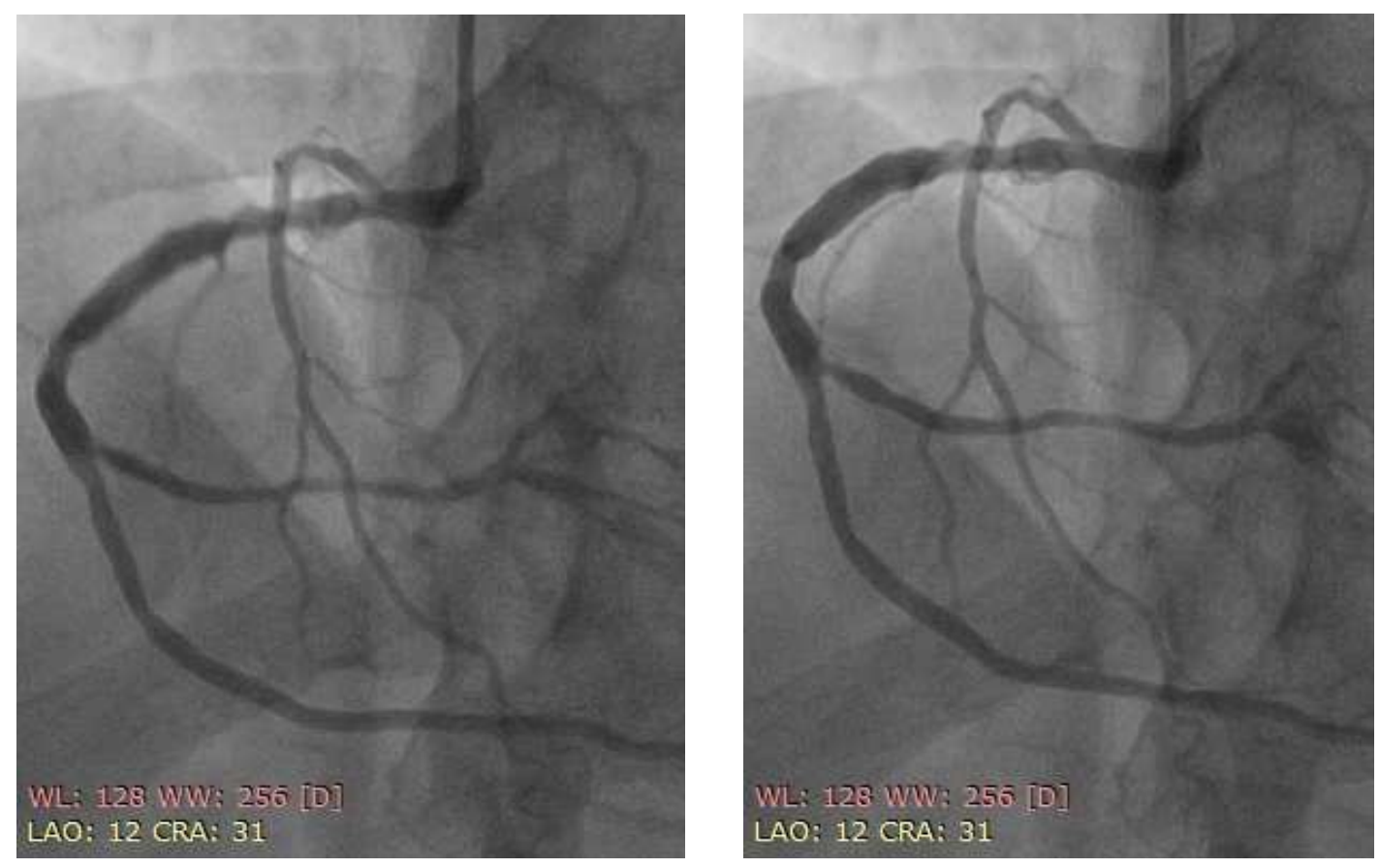

Figura 3. Exemplo de imagens Fluoroscópicas clínicas do coração. Na esquerda há maior borrosidade cinética na imagem e na direita a redução do tempo de exposição melhorou a sua qualidade.

O emissor de radiação de um equipamento de fluoroscopia tem características diferentes do emissor de um equipamento de radiografia convencional. Este tipo de emissor de radiação precisa operar em modo contínuo durante longos períodos de tempo, da ordem de horas, com tensões na faixa de 70 a $100 \mathrm{kV}$, correntes de $50 \mathrm{~mA}$ e imagens com frequência de 15 quadros por segundo, num intervalo de tempo total que pode variar de 20 a 100 minutos (ICRP, 2013).

Em regime de fluoroscopia, um fator importante a ser avaliado é a capacidade térmica do conjunto: anodo do tubo, cúpula, sistema de bomba de óleo e irradiador de calor, uma vez que este gera a radiação necessária e muito calor como parte do processo, o qual deve ser 
dissipado em tempo curto para não aumentar ainda mais a duração do procedimento. $\mathrm{O}$ sistema de refrigeração precisa retirar o calor gerado na produção de radiação, de forma eficiente, permitindo o uso prolongado do conjunto de maneira que a temperatura do alvo não atinja valores próximos à sua temperatura de fusão. O colimador é parte integrante do conjunto emissor de radiação, seja o colimador de contorno cardíaco ou os colimadores para arteriografias de membros inferiores, os quais adquirem função de restringir o campo radiativo e atenuar parcialmente o feixe de radiação, sobre regiões a serem protegidas no paciente. Esses colimadores especiais foram desenvolvidos para minimizar o problema das diferenças de atenuação das estruturas do tórax do paciente, que ocupam uma faixa enorme de atenuações, exigindo igualmente respostas adequadas às exposições do detector, e assim possibilitar a formação de imagens de boa qualidade, desde as imagens correspondentes ao pulmão até as das regiões do coração superpostas à coluna. O colimador tem ainda a função de evitar que o conjunto emissor de radiação se aproxime muito da pele do paciente, e consequentemente possa causar danos pelo rápido aumento da dose na pele quando a distância for muito reduzida. O regulamento vigente estipula que a distância mínima a ser aplicada é de $38 \mathrm{~cm}$ (do ponto focal à entrada da pele, Distância Foco-Pele, DFP (MS / ANVISA, 1998, 2005) Outros componentes que também fazem parte de um equipamento de fluoroscopia são: mesa para o paciente, detector de imagem e estrutura mecânica de suporte ao emissor e ao detector. A Figura 4 mostra um exemplo de equipamento moderno de fluoroscopia, equipado com detector intensificador de imagens e com Controle Automático de Exposição (Automatic Exposure Control, AEC).

Quanto ao regime de registro instantâneo de imagens, este não é o quesito de maior exigência para a fluoroscopia comumente utilizada em centro cirúrgico e conhecida como arco cirúrgico. Já no caso de equipamentos de cardiologia intervencionista e angiografia por subtração digital, há a necessidade da aquisição de um grande número de imagens com maior quantidade de informações diagnósticas. Neste caso, com o equipamento operando em regime de radiografia, há a necessidade de maior exposição por imagem para melhorar a relação sinal-ruído. Isto deve-se ao fato de que estas imagens servirão para análise posterior pelo médico, que buscará detectar lesões sutis que não foram detectadas durante o procedimento na sala do exame.

Os equipamentos de angiografia cardíaca ou de subtração digital são empregados para duas finalidades: a) para diagnóstico, onde há necessidade de fluoroscopia e de documentação; b) para procedimento terapêutico (exemplo angioplastia coronária) 
emitindo radiação por intervalos maiores de tempo, com intervalos superiores a 30 minutos para um paciente.

\section{Intensificador de Imagens (II)}

Com o desenvolvimento do II, foi possível resolver alguns dos problemas que preocupavam os pesquisadores da época: o brilho reduzido do écran passou a ser um brilho mais intenso, dispensando assim a necessidade da visão noturna para avaliar as imagens. Isto trouxe benefício imediato quando se considera o aspecto isolado da visão e que agora emprega os cones na retina, que é mais rica em detalhes do que a visão noturna. Outro problema resolvido foi a possibilidade de desenvolver um dispositivo que monitorasse o brilho na saída do intensificador de imagens e utilizasse esta informação para controlar os parâmetros de exposição do gerador de raios X (Geise, 2001). Este dispositivo foi o AEC

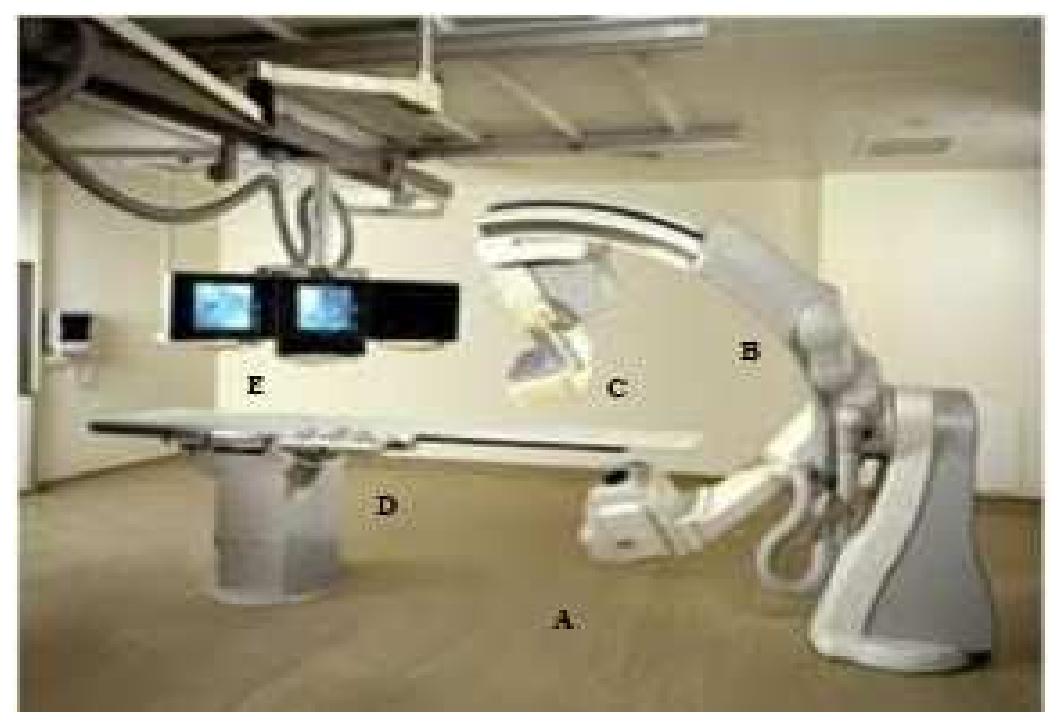

Figura 4. Equipamento atual de fluoroscopia com: a) o tubo de raios $\mathrm{x}$; b) o arco em C; c) o detector FP; d) a mesa de procedimentos e e) os monitores. (http://www.protomedlabs.com/wpcontent/uploads/2012/04/Interventional-Cardiology-Equipment-11-288x230.jpg acessado em 20/08/2013). 


\section{Controle Automático de Exposição (AEC)}

O equipamento de fluoroscopia está equipado com o dispositivo de ajuste automático de exposição ou de brilho, AEC ou ABC (Automatic Brightness Control) que consiste de um sensor na saída do II para manter a Taxa de Exposição no Detector de Entrada da Imagem ,TEEDI, constante e com isso, também constante o brilho na saída do II, dispensando a necessidade de reajustes durante o procedimento e evitando doses desnecessariamente elevadas ou evitáveis (Balter, 2006). Caso o equipamento esteja equipado com detector FP, o automatismo da exposição será feito a partir do controle dos valores de pixels numa região definida no detector para esta finalidade. Como este é um ajuste de configuração interna do equipamento, que depende do fabricante, o físico precisa de ferramentas quantitativas para ter argumentos mais convincentes e conseguir que o fabricante mude os ajustes de fábrica. O problema aqui é que os ajustes são configurados na fábrica, considerando as preferências médias dos médicos e sem dar a chance ao médico que será o usuário final de optar por ajustes diferentes. O ajuste feito na fábrica tem como objetivo deixar o cliente satisfeito, com valores de taxa de exposição no detector que estarão relacionados às doses absorvidas pelos pacientes, de forma a diminuir as chances de receber reclamações dos médicos, que por sua vez também querem imagens sem ruído ou granulação que serão apresentadas a outros profissionais e que poderiam associar a granulação à má qualidade ou baixo desempenho do equipamento (den Boer et al, 1994; Balter, 2006). Neste ponto há ainda outro aspecto no papel do físico em medicina, que é o de informar os usuários, médicos e técnicos, quanto aos valores praticados de TEEDI em duas situações interessantes: a) imagem sem ruído gerada com maior TEEDI e b) imagem com pouco ruído e TEEDI menor, chamada de otimizada. A partir destas informações, este deve criar uma consciência na equipe de que se deve buscar valores mais baixos da TEEDI, tolerância do médico a valores mais baixos de TEEDI, com a condição de que a qualidade dos serviços médicos não seja prejudicada ou que os benefícios ao paciente sejam maximizados com doses tão baixas quanto exequível, ou seja, o princípio ALARA (ICRP, 1991). A escolha por valores mais baixos da TEEDI para imagens adequadas ao diagnóstico é também condição de redução da exposição da equipe às radiações $X$, uma 
vez que em cardiologia intervencionista a equipe trabalha dentro da sala, ao lado do paciente e que, se a TEEDI é menor, consequente haverá redução do espalhamento, com os outros parâmetros mantidos constantes (Lin e Rauch, 2012; Faulkner et al, 1998). A tomada de decisão pela equipe é então conhecida como decisão informada sobre a dose ao paciente e à equipe. Autores têm chamado a atenção para este aspecto do problema: a necessidade de treinamento das equipes de médicos e técnicos para atualização de conceitos por parte das equipes de médicos e técnicos que utilizam a fluoroscopia (Vano, et al, 2005; Geise, 1999).

\section{Detectores Flat Panel (FP)}

O uso do detector II não foi unânime, provavelmente pelo seu alto custo. No ano de 1998 ainda havia fabricantes de fluoroscopia convencional no Brasil, quando ocorreu a publicação do regulamento do Ministério da Saúde (MS / ANVISA, 1998), que definiu um prazo a partir do qual seria proibida sua comercialização, instalação e transferência de um local a outro. O objetivo desta proibição foi o de reduzir as doses evitáveis à população. É interessante observar, porém, que em alguns países este tipo de tecnologia ainda é utilizado, como a China. A partir de 2000 surgiu uma nova alternativa como detector de fluoroscopia, o Flat Panel, FP. Desde então, o II está sendo substituído gradativamente por esses novos detectores. A Figura 5 ilustra o detector FP. O FP consiste de um painel fluorescente de cristal de CsI que é acoplado a uma matriz de fotodiodos que detectam o padrão de intensidades de radiação. O padrão de intensidades é transformado em luz no CsI, o qual é posteriormente transformado em sinal elétrico nos fotodiodos. Estes sinais elétricos são convertidos em imagem digital, no conversor Analógico Digital, e esta imagem é transferida diretamente à memória do computador, como mostra a Figura 6. A comparação do desempenho dos dois detectores é ainda complexa pelo alto número de variáveis presentes (Tapiovaara, 2006). Os dois detectores apresentam aspectos positivos e negativos de forma equilibrada quanto aos seus resultados. Ambos são sensíveis o suficiente para a fluoroscopia, sendo que para o FP há potencial para redução da TEEDI. O contraste do detector FP é melhor que o do detector II, pois neste último há a presença do veiling glare (Seibert et al, 1984; Baba et al, 2002), que interfere negativamente no seu 
desempenho quando o desafio é detectar pequenas diferenças de atenuação. No FP o espalhamento é reduzido devido a estrutura em forma de agulha dos seus cristais de CsI (Nickoloff e Strauss, 2011), A detectabilidade de contraste ou o limiar de detecção de contraste do detector FP é portanto melhor que a do detector II (Davies et al, 2007). A resolução do detector II é melhor que a do detector FP pois como tem a função de magnificação a resolução é melhor do que no FP, onde o pixel tem dimensões físicas definidas, da ordem de 0,18 mm, sendo chamado de pitch do detector (Giger e Doi, 1986; Jiang e Wilson, 2006)). Em situações onde a exposição deve ser a mínima possível, é possível agrupar alguns pixels do detector FP para aumentar a colecção de fótons e conseguir maior sensibilidade ao conjunto. Entretanto, o emprego do binning traz aumento da sensibilidade, mas também queda na resolução, pois aumenta as dimensões do pixel. Caso fosse construído um detector com pixel menor, isto criaria uma situação inversa: teria a vantagem de melhorar a sua capacidade de detectar detalhes menores, mas o preço seria a redução da detecção de fótons (pixels menores) com o aumento do ruído e a necessidade de aumento da exposição, o que comprometeria a detectabilidade das estruturas de baixo contraste (Vano et al, 2005). Esses exemplos têm o objetivo de ilustrar a complexidade de tomar decisões num ambiente onde muitas variáveis interferem nos resultados e explica o por quê foram necessários anos de esforços em pesquisa.

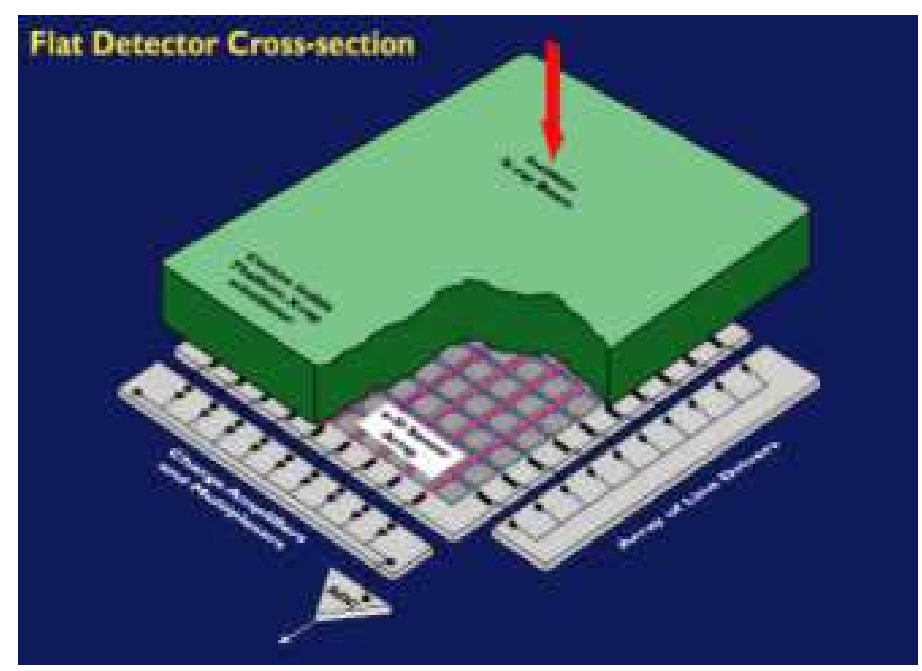

Figura 5. O detector FP e suas partes principais. (http://lxi.leeds.ac.uk/research/past/fd/ acessado em 20/08/2013). 


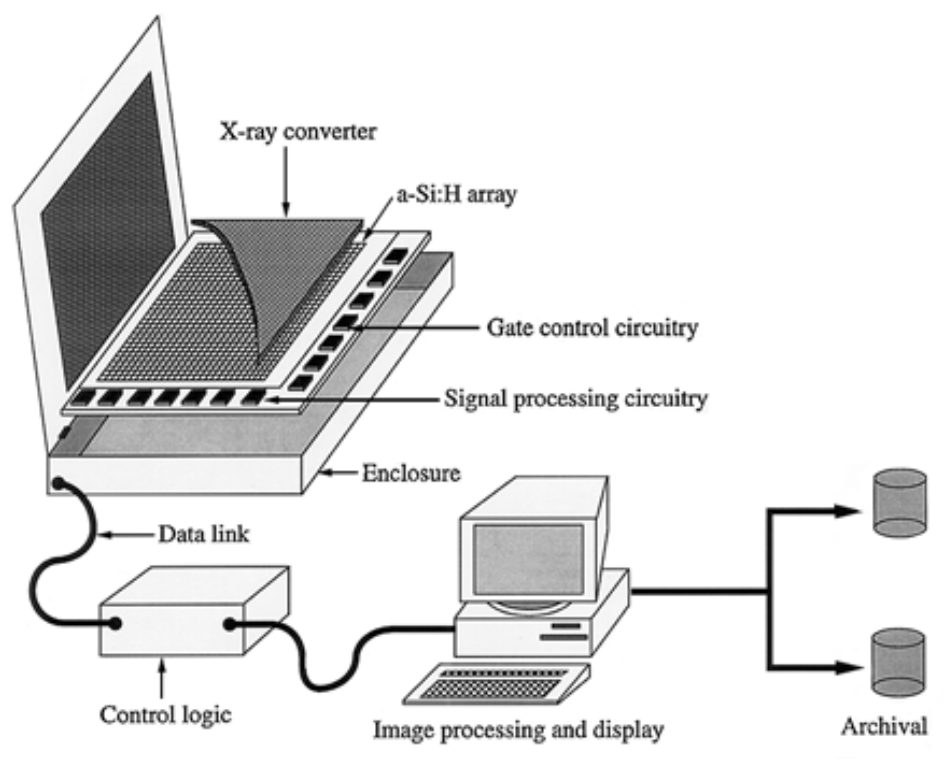

Figura 6. Detector flat panel utilizado em fluoroscopia com a unidade de controle lógico, a unidade de processamento e apresentação da imagem e a unidade de arquivo.

(http://www.aps.org/publications/apsnews/199607/advances.cfm acessado em 20/08/2013).

Ainda comparando as características dos dois detectores, o II tem grandes dimensões e depende de tensões de focalização para fornecer imagens com resolução superior à do FP, mas sujeitas a variações pelo desgaste de componentes e variações típicas das fontes de alimentação, gerando a necessidade de reajustes regulares do foco dos intensificadores. O FP não apresenta este problema, mas apresenta a desvantagem de ao longo do tempo possuir os dead pixels. Estes são pontos cegos que não enviam informação ao sistema. Os programas de pós-processamento da imagem procuram correções para este problema, mas por mais que tentem resolvê-lo, não é possível recuperar uma informação que não existe mais. Com isto, é feito uma interpolação utilizando os dados de pontos vizinhos e um algoritmo de interpolação para estimar o valor para os dead pixels. Devido a esta situação, existem as seguintes dúvidas no caso de haver uma maior concentração de pontos cegos numa determinada área: qual seria a tolerância para o número de dead pixels e a partir de que limiar o detector deveria ser substituído. Na Figura 7 é apresentada uma imagem do detector FP sem as correções do pós-processamento da imagem (Balter, 2006). 


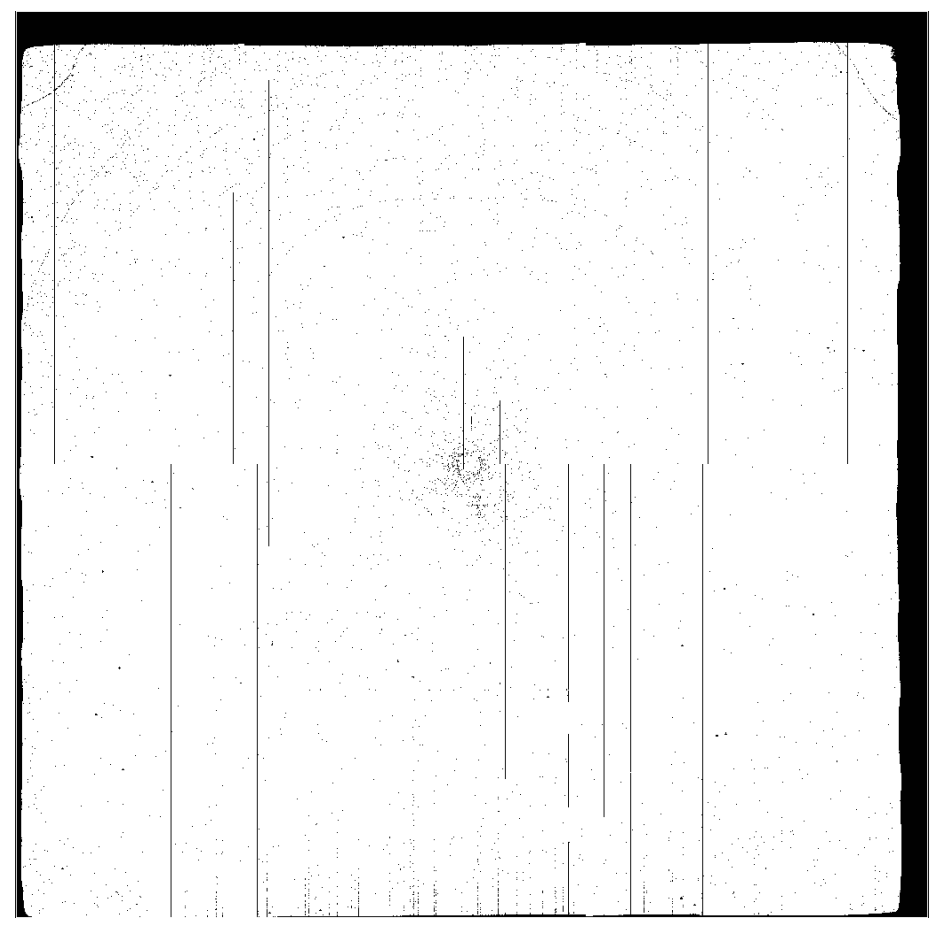

Figura 7. Imagem RAW de detector Flat Panel apresentando os pontos sem sinal ou dead pixels (Colbeth et al, 1998).

\section{Aplicações da fluoroscopia}

A fluoroscopia é comumente aplicada em procedimentos de estudo das estruturas internas do paciente, para fins diagnósticos e terapêuticos, através de intervenções minimamente invasivas que expõem o paciente a riscos inferiores aos de um procedimento cirúrgico. A maioria dos procedimentos de cardiologia intervencionista ocorre com o paciente consciente, durante todo o procedimento.

Exemplos de aplicações: a) sistema digestório (deglutição, peristaltismo do esôfago, estômago e intestinos); b) sistema circulatório (angioplastias e embolizações de artérias que alimentam tumores e aneurismas em vasos sanguíneos do cérebro, coração e pulmões, abdôme e extremidades); c) traumatologia: restaurar movimentos do esqueleto, ossos e os efeitos de traumas e de terapias; d) urologia (rins e ureteres e bexiga, presença e movimento de cálculos); e) localização de campos de radioterapia guiada por imagens, em tempo real (durante o tratamento há movimento dos órgãos dentro do campo de radiação) e outros. 


\section{Cardiologia Intervencionista}

Os procedimentos de cardiologia intervencionista podem ser de diagnóstico ou terapêuticos. Os intervalos de tempo de procedimento de diagnóstico são menores e representam um desafio importante para o equipamento. $\mathrm{O}$ tubo de raios $\mathrm{X}$ precisa produzir pulsos de radiação com milésimos de segundo de duração e de até $70 \mathrm{~kW}$ de potência, com a necessidade de produzir imagens de qualidade de artérias da região do tórax e do coração, que se movem à velocidades da ordem de $10 \mathrm{~cm} / \mathrm{s}$. Esses equipamentos vêm evoluindo bastante desde a década de 80 , quando houve um grande crescimento dos procedimentos intervencionistas, impulsionados pelo interesse geral em evitar os riscos de uma cirurgia cardíaca aos pacientes. O paciente deve ficar na mesa de procedimentos por uma hora e em alguns casos, por mais tempo, dependendo da complexidade do caso. Alguns pacientes apresentaram efeitos determinísticos como lesões de pele pósprocedimento, o que tem despertado a atenção da comunidade de físicos em medicina, para a necessidade de melhorar os métodos de medição da taxa de exposição que está diretamente relacionada à dose absorvida, para alertar os cardiologistas e intervencionistas em geral, sobre os valores dessas a que o paciente está sendo exposto (Balter, 2006).

\section{Estado de Otimização}

A qualidade dos serviços médicos prestados ao paciente depende fundamentalmente da qualidade da imagem e da taxa de exposição. A dose absorvida pelo paciente também é um aspecto importante na cardiologia intervencionista por se tratar de valores da ordem de 3 a 5 Gy (ICRP, 2013), como ilustra a Figura 8. Considerando a complexidade do equipamento e dos procedimentos que serão realizados neste equipamento, o problema adquire proporções de grande complexidade. A especialidade está entre as que utilizam maiores exposições de raios $\mathrm{X}$, para o paciente e para a equipe. O problema central para os físicos em medicina é procurar reduzir as doses, indo contra a 
qualidade da imagem, pois o detector precisa de sinal forte para gerar imagens de qualidade. O compromisso pode ser atingido quando se conhece bem os fenômenos envolvidos no processo e há uma métrica adequada para avaliar os ganhos, em termos de doses e em termos de qualidade. Em termos de doses, a taxa de exposição normalmente utilizada pelo fabricante é a taxa de exposição na entrada do detector.

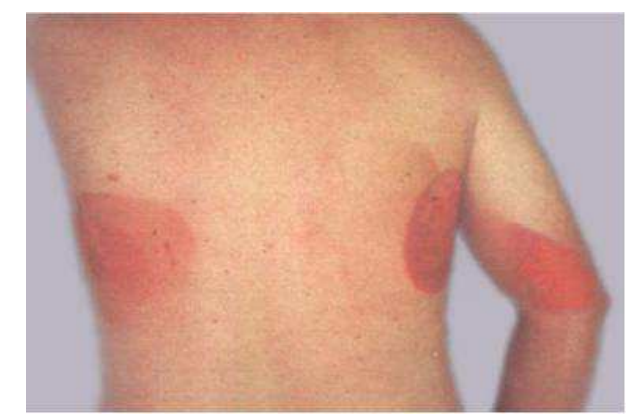

Figura 8. Paciente apresentando eritema radio-induzido pós procedimento de cardiologia intervencionista, onde a área afetada no braço direito é exemplo de dose evitável (MS / ANVISA, 1998; ICRP, 2013; Canevaro, 2009; Krain, 1992; Lemoigne et al, 2009).

\section{Avaliação da qualidade}

Em termos de quantificar a qualidade da imagem, o objeto simulador chamado Contraste/Detalhe permite expressar a qualidade de forma quantitativa, apesar de ainda precisar de uma avaliação subjetiva da imagem por observador humano. Este método tem sido amplamente utilizado em levantamentos de qualidade e comparações de desempenho entre diferentes equipamentos. A Figura 9 apresenta uma ilustração da imagem obtida com o Objeto Simulador do tipo Contraste Detalhe. 


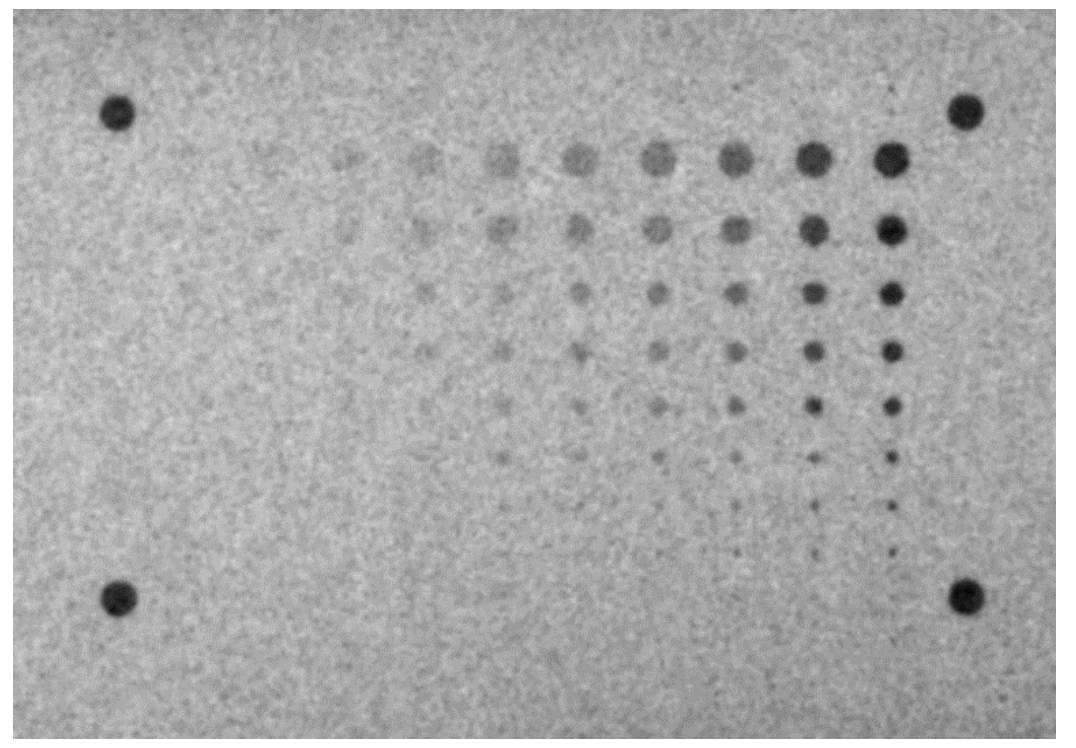

Figura 9. Imagem do objeto simulador CD DENT para avaliar os parâmetros limiar de contraste e limiar de detalhe (http://www.nuclemed.be/product.php?cat=124\&prod=529 acessado em 20/08/2013).

O processo para um diagnóstico radiológico se dá pelas seguintes etapas: O médico analisa a imagem para diagnóstico de lesões causadas por exemplo, por patologias. O tratamento destas patologias tem maior chance de cura, quando o diagnóstico for feito em estágios iniciais da patologia. Isto exige que o sistema que gera imagens diagnósticas, tenha condições de detectar lesões pequenas, que apresentam pequena atenuação da radiação e conhecidas por estruturas de baixo contraste. $\mathrm{O}$ mesmo ocorre quanto ao detalhe. O sistema deve ter capacidade de documentar estruturas muito pequenas. Para avaliar essas duas características, utiliza-se um objeto simulador de lesões em estágios iniciais, o objeto simulador Contraste/Detalhe, que pode ser construído com diferentes materiais, como alumínio, cobre (ou como desenvolvido neste trabalho de acrílico). O dispositivo apresenta furos circulares com o mesmo diâmetro e diferentes profundidades nas linhas e furos circulares com mesma profundidade e diâmetros diferentes nas colunas. Ao se produzir imagens deste dispositivo, o resultado será a visibilidade parcial dos furos, pois o sistema conseguirá detectar os furos a partir do seu limiar de detecção de contraste. O número de furos visíveis nas linhas é uma medida de quão bem o sistema executa esta tarefa de detectar lesões sutis, de baixo Contraste. Os furos dispostos nas colunas é uma medida do menor Detalhe que o sistema consegue detectar. Os dois resultados, numéricos, 
informam a capacidade do sistema de detectar lesões sutis e o produto desses dois resultados é chamado de figura de mérito ou FOM (Figure Of Merit) (Thomas et al, 2005).

Ao buscar a otimização do processo de produção de imagens para diagnóstico, uma ação comum é tentar reduzir a taxa de exposição. Este ajuste pode trazer aumento do ruído na imagem e afetar negativamente a visualização das estruturas de baixo Contraste e pequenos Detalhes. As imagens do objeto simulador fornecem uma referência para as decisões: qual o nível mais adequado para a taxa de exposição, que seja baixo para minimizar as exposições do paciente e da equipe, mas que seja bom para não interferir na detecção das lesões sutís pelo médico (Maolinbay et al, 2000; Marshall et al, 1992)

O quadro fica mais complexo quando se introduz outras variáveis que interferem no processo: o movimento do objeto, no caso clínico o coração por exemplo, que se move a $10 \mathrm{~cm} / \mathrm{s}$; a espessura do paciente, que em cardiologia pode chegar a $35 \mathrm{~cm}$, entre outros parâmetros. Há ainda a possibilidade do físico comparar o desempenho de equipamentos diferentes e avaliar em que estágio de otimização encontra-se cada equipamento ou cada processo, pois se trata de equipamentos com diferentes tempos de serviço, data de fabricação, tecnologias, origem e durabilidade intrínseca. Atualmente no Brasil existem em funcionamento equipamentos fabricados na Europa, Ásia e Américas e cada fabricante busca soluções engenhosas para o desafios já citados. A comunidade de físicos em medicina tem contribuído com melhorias dos protocolos de medições de radiações e medições mais objetivas da qualidade da imagem. Em condições controladas de laboratório tem sido possível utilizar técnicas quantitativas e objetivas muito sofisticadas, mas que se revelaram demoradas e/ou dependentes de recursos não disponíveis em hospitais (Gagne et al, 2006) e o uso de objetos simuladores tem sido prática frequente no radiodiagnóstico para avaliar o desempenho de sistemas geradores de imagens como o equipamento de cardiologia intervencionista (Cowen et al, 1987; Marshall et al, 2001). 


\section{Capítulo3}

\section{Materiais e Métodos}

\section{Materiais}

Neste trabalho um protocolo específico foi derivado do sugerido no Report \#74 da AAPM (Shepard e Lin, 2002), do trabalho de Boone (Boone, 1993), de Burgess (Burgess, 1999), de Bath (Bath, 2010) do Marshall (Marshall et al, 2010) e no Manual de Segurança Radiológica (MS / ANVISA, 2005) uma vez que estes são mais adequados para servirem como base para as medições da qualidade da imagem (Contraste e Detalhe ou Resolução) e da exposição no detector do equipamento de fluoroscopia cardíaca. O protocolo desenvolvido consta dos seguintes testes: a) conferência da tensão aplicada ao tubo de raios X e da medida da taxa de exposição no detector; b) obtenção das amostras das imagens fluoroscópicas; c) avaliação do detalhe e do contraste das imagens obtidas.

No protocolo obtido, a avaliação da tensão aplicada ao tubo deve estar em $(80 \pm 5)$ $\mathrm{kV}$ e para a avaliação do detalhe foi considerado o menor furo visível não deve ser maior do que 1,3 mm de diâmetro (dos furos feitos no acrílico) e para a avaliação do contraste o limiar inferior de detecção do sensor (II ou FP) não pode ser maior do que $10 \%$, das porcentagens apresentadas para contraste no dispositivo de teste.

Para a avaliação das taxas de exposição e da qualidade da imagem fluoroscópica cardíaca foram investigados inicialmente em 40 equipamentos, dos quais foram selecionados 19 segundo um critério baseado em seu uso, dimensão do detector e recursos do gerador.

Quanto ao uso: o equipamento deve ter uso exclusivo em cardiologia intervencionista ou estar a ela dedicado $70 \%$ do seu tempo de utilização.

Quanto às dimensões do detector: este deve ter pelo menos $18 \mathrm{~cm}$ de diâmetro (II) ou de diagonal (FP). 
Quanto aos recursos do gerador: este deve produzir pulsos de radiação da ordem de 1 milésimo de segundo, ter faixa de voltagens pico de raios $\mathrm{X}$ de 60 a $120 \mathrm{kV}$, ter corrente máxima de tubo de $200 \mathrm{~mA}$, ter potência de $25 \mathrm{~kW}$, ter arco de posicionamento com diâmetro mínimo de $100 \mathrm{~cm}$ e estar equipado com sistema de controle automático de exposição (Automatic Exposure Control, AEC).

\section{Dispositivo para avaliação do $k V$ do equipamento}

Fabricado pela empresa UNFORS/Rad Fluoro/90620; consiste de uma caixa $(14,0$ x 7,5x $2,7 \mathrm{~cm}^{3}$ ) contendo dois fotodiodos como sensores em paralelo e posicionados sobre eles estão dois filtros de cobre com diferentes espessuras, para através da razão entre suas leituras se obter a tensão aplicada ao tubo (Figura 1).

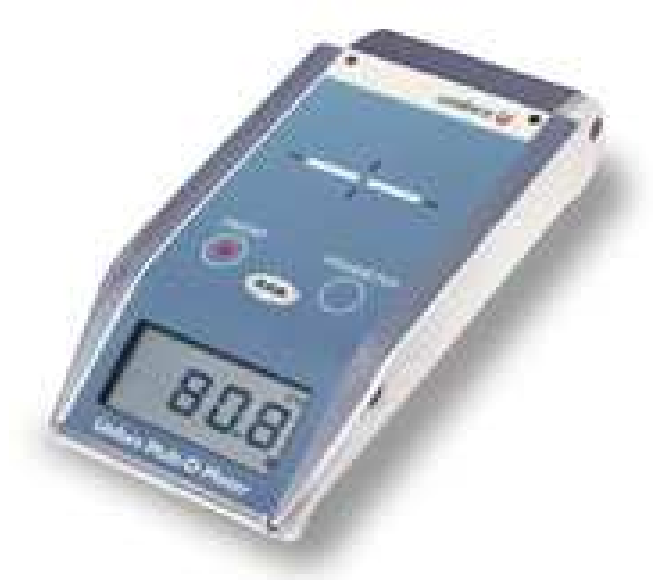

Figura 1 Imagem digital do dispositivo utilizado para medir a tensão aplicada ao tubo. 
Os 19 equipamentos selecionados para fazer parte da amostra a ser testada são provenientes de vários países: 13 da Europa (8 do país A e 5 do país B) 1 da Ásia (país C) e 5 das Américas (1 país D e 4 país E).

TABELA 1. Equipamentos de Cardiologia Intervencionista selecionados para este estudo.

\begin{tabular}{ccccc}
\hline Número & Código & Fabricante & Detector (II/FP) & Idade (meses) \\
1 & AJP & E & II & 24 \\
2 & CPP & E & II & 40 \\
3 & SBS & A & II & 22 \\
4 & S7S & C & II & 36 \\
5 & S8S & B & II & 18 \\
6 & S9S1 & B & II & 53 \\
7 & S9S2 & B & II & 16 \\
8 & SPS & B & II & 40 \\
9 & SRS & A & II & 55 \\
10 & TAP & E & II & 46 \\
1 & & & & \\
2 & CPP & E & FP & 1 \\
3 & MSP & B & FP & 32 \\
4 & SAS & D & FP & 38 \\
5 & S1S & A & FP & 24 \\
6 & S2S & A & FP & 89 \\
7 & S5S & A & FP & 16 \\
8 & S6S & A & FP & 26 \\
9 & S9S3 & A & FP & 92 \\
& SRS & A & FP & 18 \\
\hline
\end{tabular}

Cada equipamento da tabela foi utilizado para as medições em todos os testes, com o conjunto de filtros (4 a 6 filtros de cobre com $0,5 \mathrm{~mm}$ de espessura, simuladores de paciente) colocado sobre o apoio do colimador do tubo de raios X e a câmara de ionização em um suporte sobre a mesa, a $65 \mathrm{~cm}$ do ponto focal do tubo e a $25 \mathrm{~cm}$ da entrada do Detector de Imagens (FP ou II). A tensão aplicada ao tubo foi ajustada no programa de Cardio de forma automática pelo AEC, ficando entre 75 e $85 \mathrm{kV}$, com frequência de aquisição de 15 imagens por segundo. A espessura total dos filtros foi dependente do ajuste 
de tensão da máquina para ficar em $(80 \pm 5 \mathrm{kV})$, como preconizado no protocolo desenvolvido.

A câmara de ionização foi utilizada para as medições da Taxa de Exposição $(\mathrm{C} / \mathrm{kg} / \mathrm{min}$ ) na entrada do detector de Imagem (TEEDI). Os valores medidos a $25 \mathrm{~cm}$ foram corrigidos pela Regra do Inverso do Quadrado da Distância também para a posição da entrada do detector. Após a medição da TEEDI, a câmara de ionização foi retirada e foi colocado em seu lugar, na mesma posição, o Objeto Simulador e foram produzidas as imagens para avaliação do desempenho do sistema, mantendo-se constantes os filtros selecionados.

\section{Características dos Sistemas de Imagem dos equipamentos selecionados}

Os Sistemas de Imagem são compostos por seus detectores e programas de processamento, que transferem as informações coletadas pelo detector para uma central de processamento que realiza operações de filtração de ruído, correção de artefatos em geral e transferência do sinal para dispositivos de apresentação das imagens finais, os monitores, nos quais os operadores das máquinas ou os cardiologistas avaliam a imagem para tomar decisões quanto ao procedimento.

Para a medição da taxa de exposição no detector foi utilizada uma câmara de ionização Keithley/96020A/17558 acoplada a um eletrômetro Keithley/35050/18515. As características da câmara de ionização $(\mathrm{CI})$ são: volume sensível $150 \mathrm{~cm}^{3}$, potencial de colecção de cargas $100 \mathrm{~V}$, faixa de energia dos feixes de raios $\mathrm{X}$ de 30 a $200 \mathrm{keV}$; calibrada através de intercomparação com padrão terciário, este por sua vez, calibrado no laboratório de calibração de instrumento do Instituto de Pesquisas Energéticas Nucleares IPEN-CNEN (Keithley, 1975). 


\section{Objeto Simulador (OS)}

Para a avaliação do desempenho do detector de imagens, em termos de detalhe e contraste das imagens obtidas, foi construído um objeto simulador (OS) a partir de uma placa de acrílico $\left(70 \times 70 \times 4 \mathrm{~mm}^{3}\right)$ com furos dispostos na horizontal em (linhas) e na vertical em colunas. Para avaliação do contraste, em cada coluna o diâmetro de cada furo foi mantido constante (preenchida com ar) e foi variada a sua profundidade, numa escala logarítmica de 3,2 a 0,5 mm. Para a avaliação do detalhe, em cada linha a profundidade dos furos foi mantida constante (preenchida com ar) e o diâmetro foi variado numa escala logarítmica de 3,2 a 0,5 mm.. O esquema deste dispositivo, objeto simulador, é dado pelas Figuras 2 a) e b).

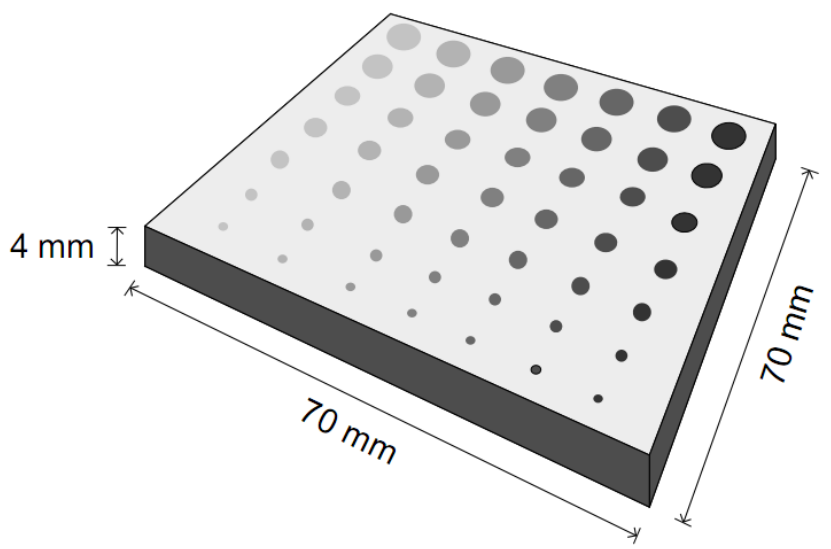

(a)

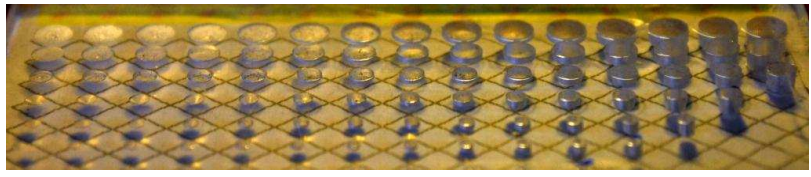

(b)

Figura 2 a) Vista superior do Objeto Simulador desenvolvido e b) foto digital da visão lateral do Objeto Simulador. 


\section{Métodos}

O filtros de cobre, para simular o paciente, foram posicionados sobre o dispositivo para a medida do $\mathrm{kV}$, que foram colocados sobre a mesa, e um feixe de raios $\mathrm{X}$, com um campo de $18 \mathrm{~cm}$ de diâmetro ou de diagonal, foi utilizado para fazer a medição que através de microprocessador e eletrônica associada apresentava o valor da tensão no visor de cristal líquido. $\mathrm{O}$ feixe de raios $\mathrm{X}$ foi acionado durante um tempo de aproximadamente 10 segundos de exposição (uma vez que o feixe da fluoroscopia tem intensidade considerada baixa para sensibilizar o sensor) e o equipamento informou a tensão aplicada ao tubo da fluoroscopia.

\section{Medida da taxa de exposição}

Utilizando o $\mathrm{kV}$ e o campo do feixe de raios $\mathrm{X}$ preconizados, os filtros simuladores do paciente sobre o colimador do equipamento e a câmara de ionização posicionada a $65 \mathrm{~cm}$ do ponto focal, o feixe foi acionado em modo cine e as medições foram realizadas para cada sistema ou equipamento. Após serem feitas as leituras no eletrômetro, suas correspondentes taxas de exposição na entrada do detector de imagem (TEEDI) foram inferidas em $\mathrm{mGy} / \mathrm{min}$.

\section{Medida da qualidade da imagem (detalhe e contraste)}

Utilizando a tensão e o campo preconizados, o $O S$ foi posicionado a $65 \mathrm{~cm}$ do ponto focal e em seguida foi acionado o feixe de raios $\mathrm{X}$, em modo cine e foram produzidas as imagens para a avaliação do contraste e do detalhe de cada sistema ou equipamento. Após a aquisição das imagens, estas foram observadas visualmente e feitas as seguintes anotações: o número de furos visíveis na linha com maior diâmetro (buracos mais rasos, 
mais acrílico e menos ar) correspondentes ao contrate e os visíveis na coluna com profundidade maior e constante e diâmetro variavel, correspondentes ao detalhe. Uma vez com os valores obtidos de cada um desses parâmetros, seu produto pode ser obtido. Deste produto se infere numericamente o nível de desempenho do sistema em termos de contraste e detalhe, o qual é identificado por FOM (Figure of Merit), sendo quanto maior o valor deste produto, maior a qualidade da imagem. 


\section{Capítulo 4}

\section{Resultados e discussões}

A partir dos materiais e das metodologias apresentados no Capítulo 3, medidas de Detalhe versus equipamento, Contraste versus equipamento, Taxa de Exposição versus equipamento e FOM versus Taxa de Exposição foram feitas para os equipamentos com dois tipos de detectores: o intensificador de imagem (II) e o flat panel (FP). Os resultados obtidos dessas medidas são apresentados a seguir juntamente com suas discussões.

\section{Detalhe x Equipamento}

Nas Figuras 1 e 2 são apresentados os resultados obtidos para o detalhe em função dos equipamentos escolhidos para o estudo, respectivamente para os detectores II e FP.

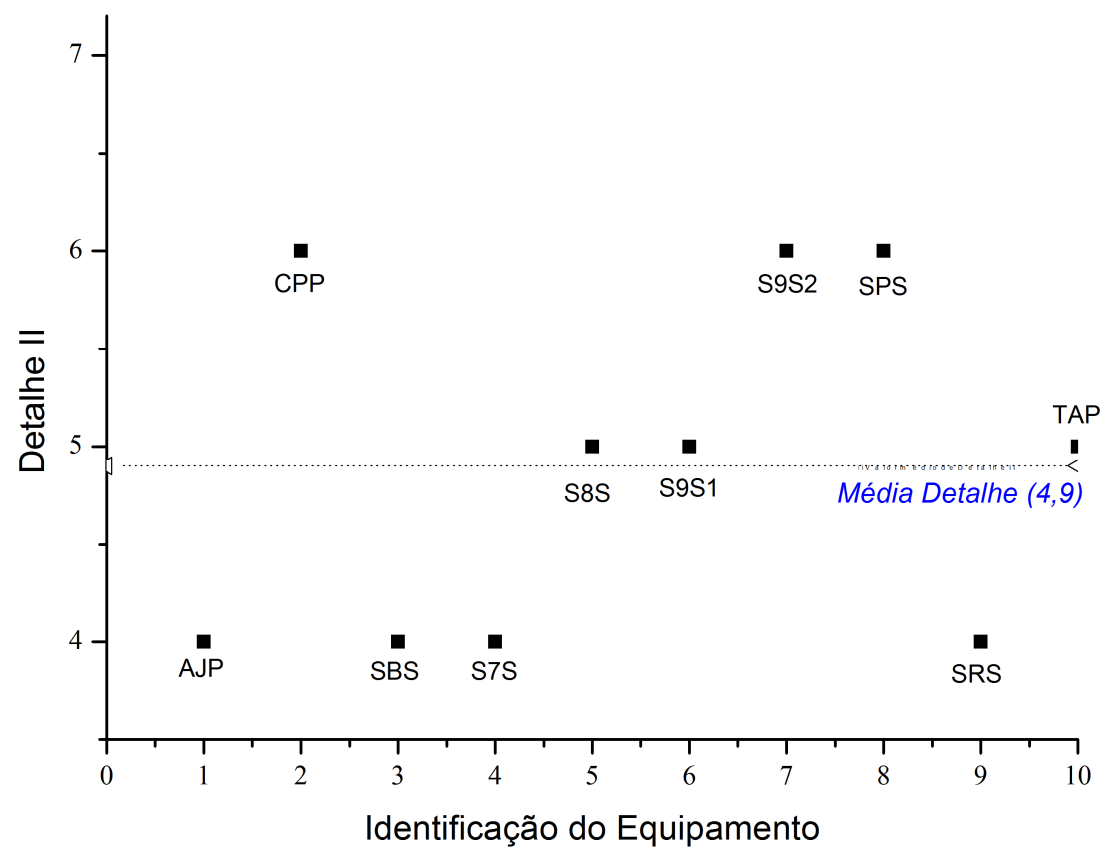

Figura 1. Detalhe II x Identificação do Equipamento, com seus respectivos códigos e o valor médio dos valores encontrados na distribuição. 
Na Figura 1 pode-se observar que seis dos equipamentos apresentaram valores de detalhe acima da média, sendo três deles com um fator de $22 \%$ e os outros três com um fator de $2 \%$. Os outros quatro valores restantes se encontram abaixo da média do grupo dos equipamentos com detectores II. Esta distribuição dos valores apresentados pode ser devida a variações, ao longo do tempo, da focalização e da tensão necessária para tal no sensor do intensificador de imagem. Outro fator que também poderia contribuir para o resultado observado, é que os detectores sendo de diferentes fabricantes foram desenvolvidos com tecnologias diferentes.

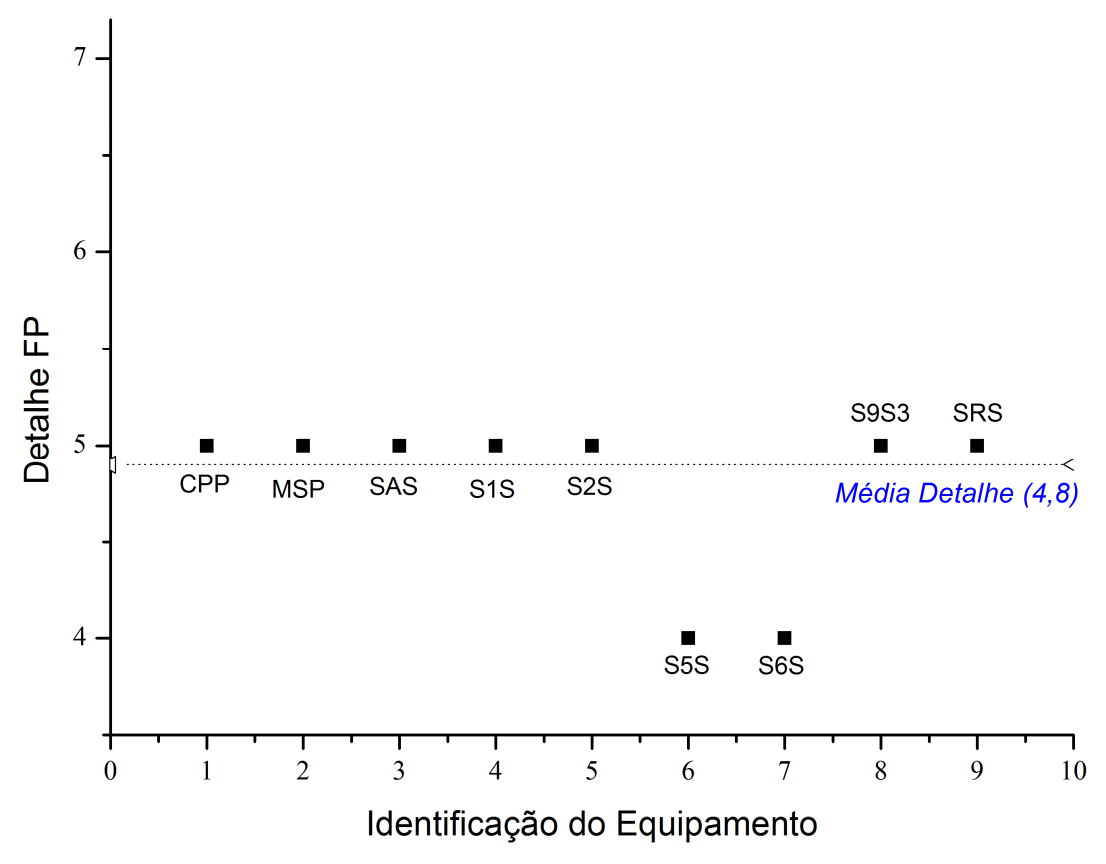

Figura 2. Detalhe FP x Identificação do Equipamento com seus respectivos códigos e o valor médio dos valores encontrados na distribuição.

Na Figura 2 observa-se que os resultados encontrados para o detector FP encontram-se, na sua maioria, próximos ao valor médio do detalhe. Na tecnologia deste tipo de detector, o tamanho do pixel, que é um fator importante na determinação do detalhe, não varia muito entre os fabricantes $(\sim 0,18 \mathrm{~mm})$, sendo esta uma possível explicação para os valores adquiridos estarem muito próximos. 
Embora as distribuições dos valores de Detalhe II e Detalhe FP sejam diferentes, seus valores médios são praticamente iguais (II 4,9; FP 4,8).

\section{Contraste x Equipamento}

Nas Figuras 3 e 4 são apresentados os resultados obtidos para o Contraste em função dos equipamentos escolhidos para o estudo, respectivamente para os detectores II e FP.

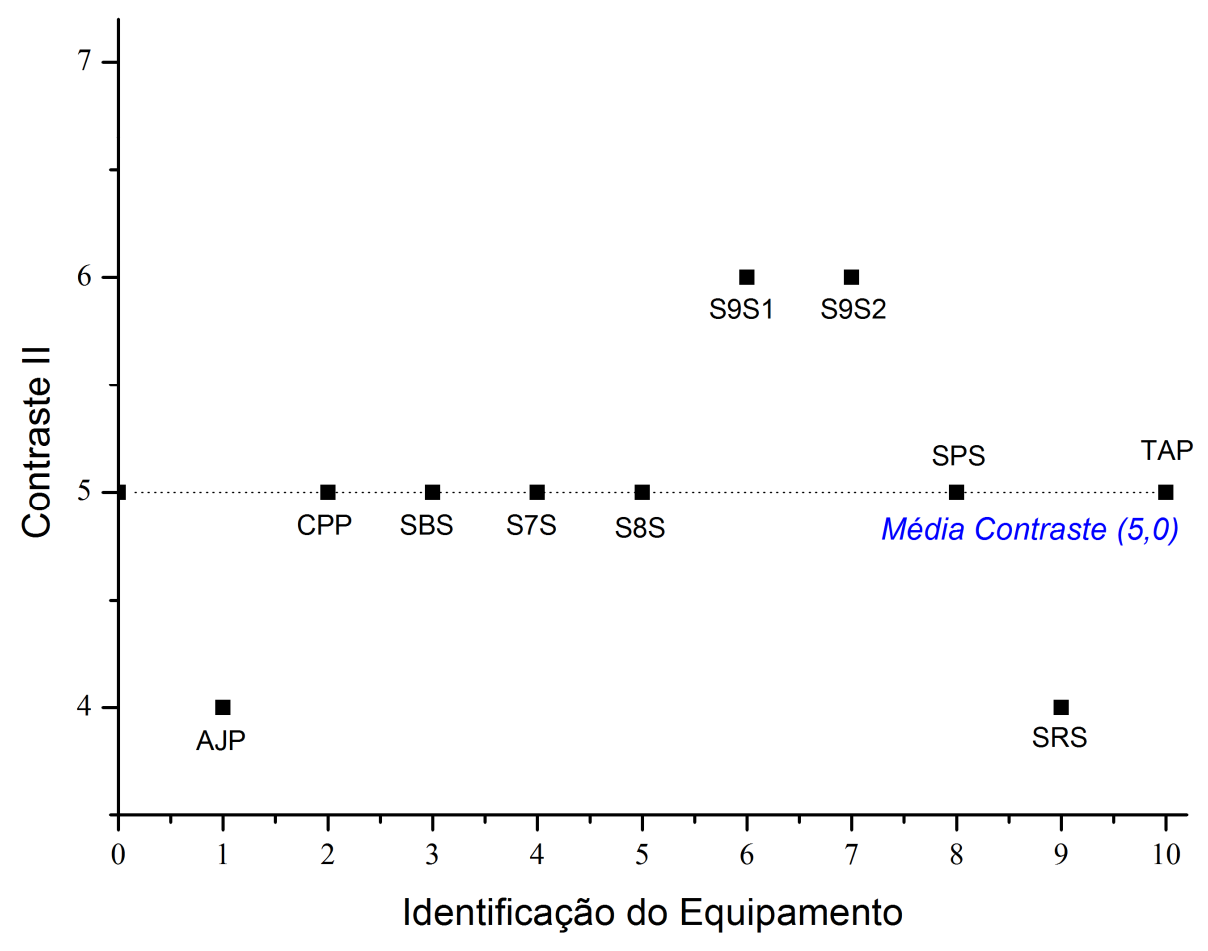

Figura 3. Contraste II x Equipamento com seus respectivos códigos e o valor médio dos valores encontrados na distribuição.

Na Figura 3 pode-se observar que dois dos equipamentos apresentaram valores de contraste acima da média, seis deles apresentaram valores na média e os dois restantes apresentaram valores abaixo da média. Esta distribuição nos valores de Contraste 
apresentados pode ser devida às variações na tecnologia utilizada na fabricação do detector II.

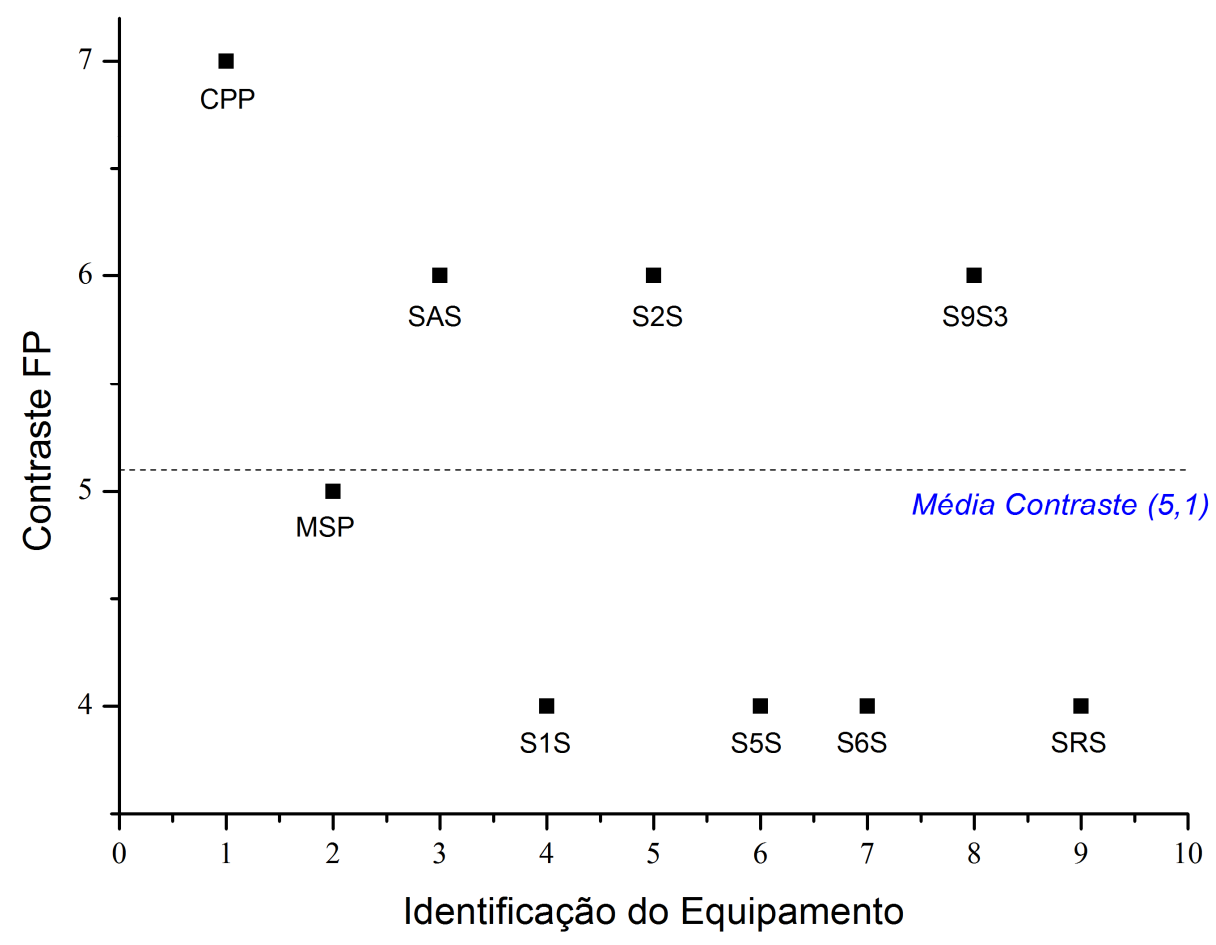

Figura 4. Contraste FP x Equipamento com seus respectivos códigos e o valor médio dos valores encontrados na distribuição.

A partir dos resultados apresentados nas Figuras 3 e 4, verifica-se que, embora seus valores de Contraste médio sejam próximos (II 5,0; FP 5,1), suas distribuições são distintas. Era esperado que o valor médio de Contraste para o Detector FP fosse maior do que o adquirido para o Detector II, primeiro porque na intensificação do brilho do Detector II ocorre um fenômeno fisicamente conhecido como "veiling glare", devido ao espalhamento de elétrons no interior do detector e que interfere negativamente no contraste; segundo porque como o princípio físico dos detectores FP é diferente e por não existir o "veiling glare", então um resultado maior para o Contraste FP seria esperado (Seibert, 1984) 
FOM x Equipamento

Nas Figuras 5 e 6 são apresentados os resultados obtidos para o FOM em função dos equipamentos selecionados para o estudo, respectivamente para os detectores II e FP.

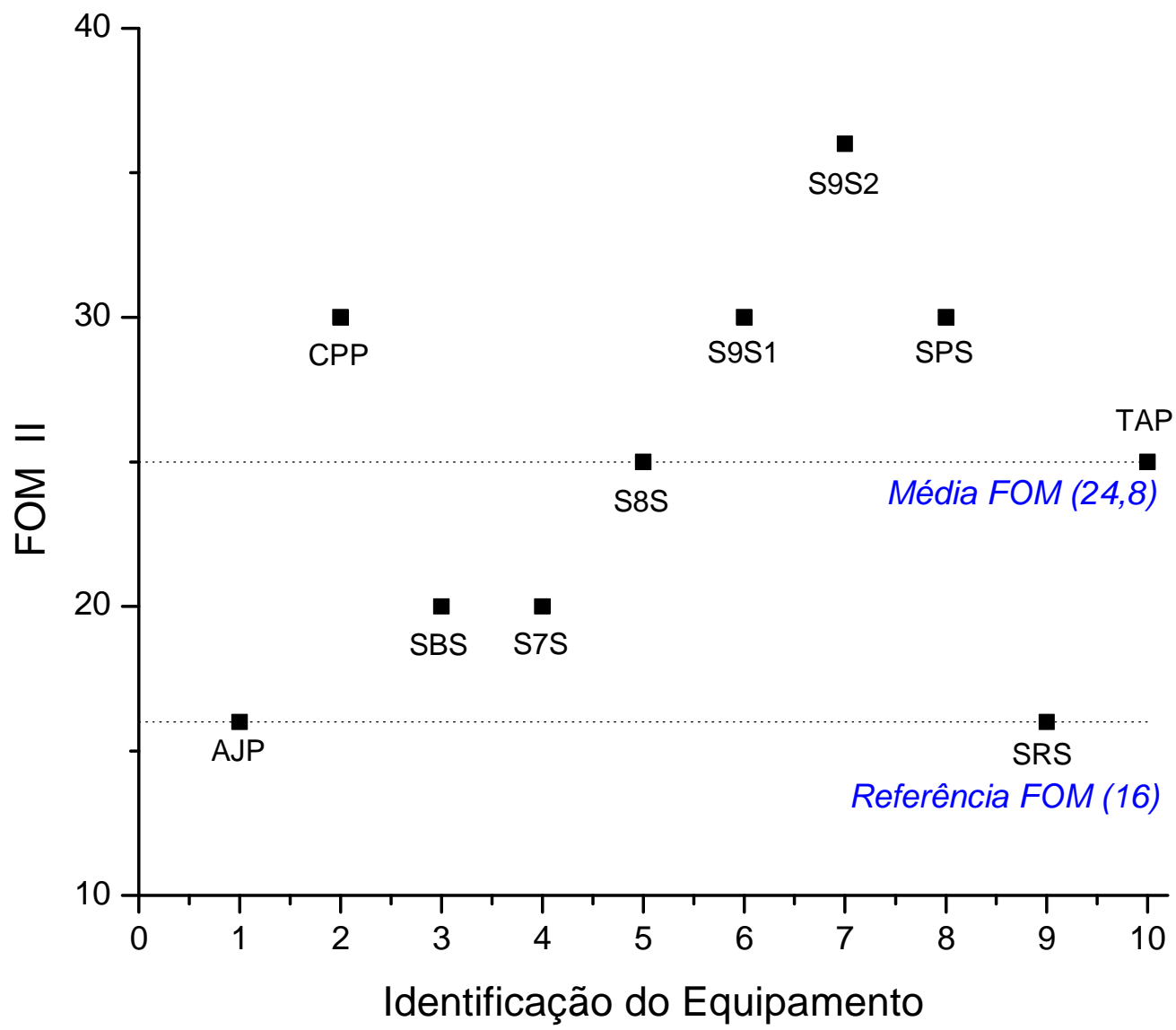

Figura 5. FOM II x Identificação do Equipamento com seus respectivos códigos e o valor médio dos valores encontrados na distribuição.

Nesta distribuição ficou evidenciada uma variação maior entre os valores menor e o maior de FOM para o detector II, uma vez que este tipo de detector apresenta variações de focalização com o tempo, devidas às variações de tensão de focalização (como na análise de Detalhe II). Com relação à média obtida, os quatro equipamentos que estão abaixo da média apresentam potencial de otimização em termos de detalhes. 


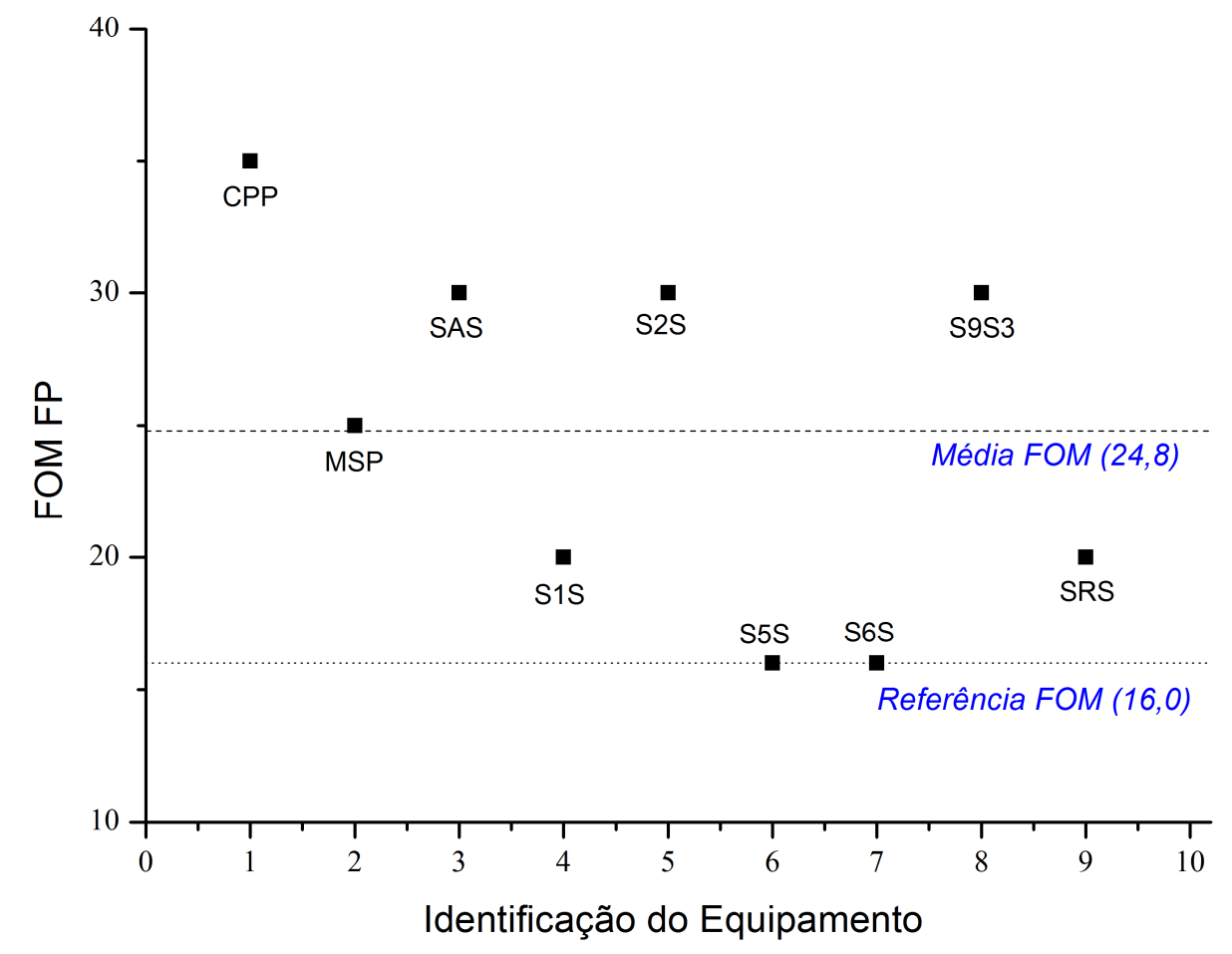

Figura 6. FOM FP x Identificação do Equipamento com seus respectivos códigos e o valor médio dos valores encontrados na distribuição.

A partir dos resultados apresentados nas Figuras 5 e 6, verifica-se que embora as duas distribuições sejam diferentes, suas médias são parecidas, e o número de equipamentos abaixo da linha média também é o mesmo. Esperava-se que devida à tecnologia do detector FP ser mais atual, o resultado da sua média seria maior do que da média para o detector II. Também nessas distribuições, tem-se quatro equipamentos que apresentam potencial de otimização do FOM em relação à média obtida. 


\section{TEEDI x Equipamento}

Nas Figuras 7 e 8 são apresentados os resultados obtidos para o TEEDI em função dos equipamentos selecionados para o estudo, respectivamente para os detectores II e FP.

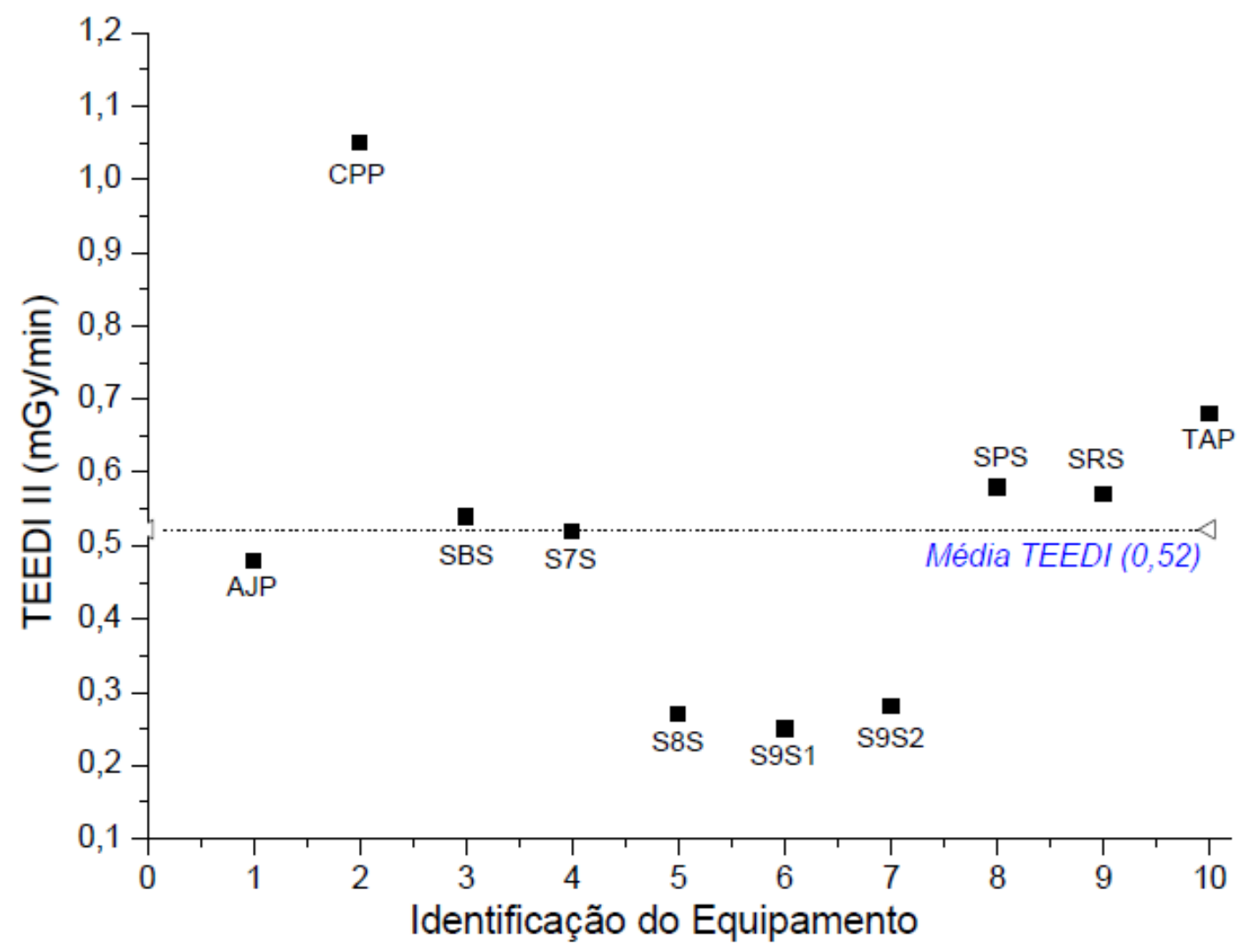

Figura 7. TEEDI II x Identificação do Equipamento com seus respectivos códigos e o valor médio dos valores encontrados na distribuição.

A distribuição encontrada para Taxa de Exposição mostrou cinco dos equipamentos acima da linha média, um na média e quatro abaixo dela. $\mathrm{O}$ equipamento 2 apresenta uma taxa de exposição muito alta e portanto tem um alto potencial de otimização. Os equipamentos 5, 6 e 7 também tem potencial de otimização da TEEDI, embora menor do que o anterior citado e os equipamentos 1 e 3 estão muito próximos da média, o que praticamente não necessitaria ajustes. Seria interessante analisar se a TEEDI do equipamento 2 fosse reduzida para o valor da média atual e uma nova média fosse obtida 
da nova distribuição, então o valor dessa nova média $(46,9)$ estaria $4,2 \%$ mais próximo do valor esperado para a média (45,0), segundo Boone et al, 1993 e Shepard e Lin, 2002.

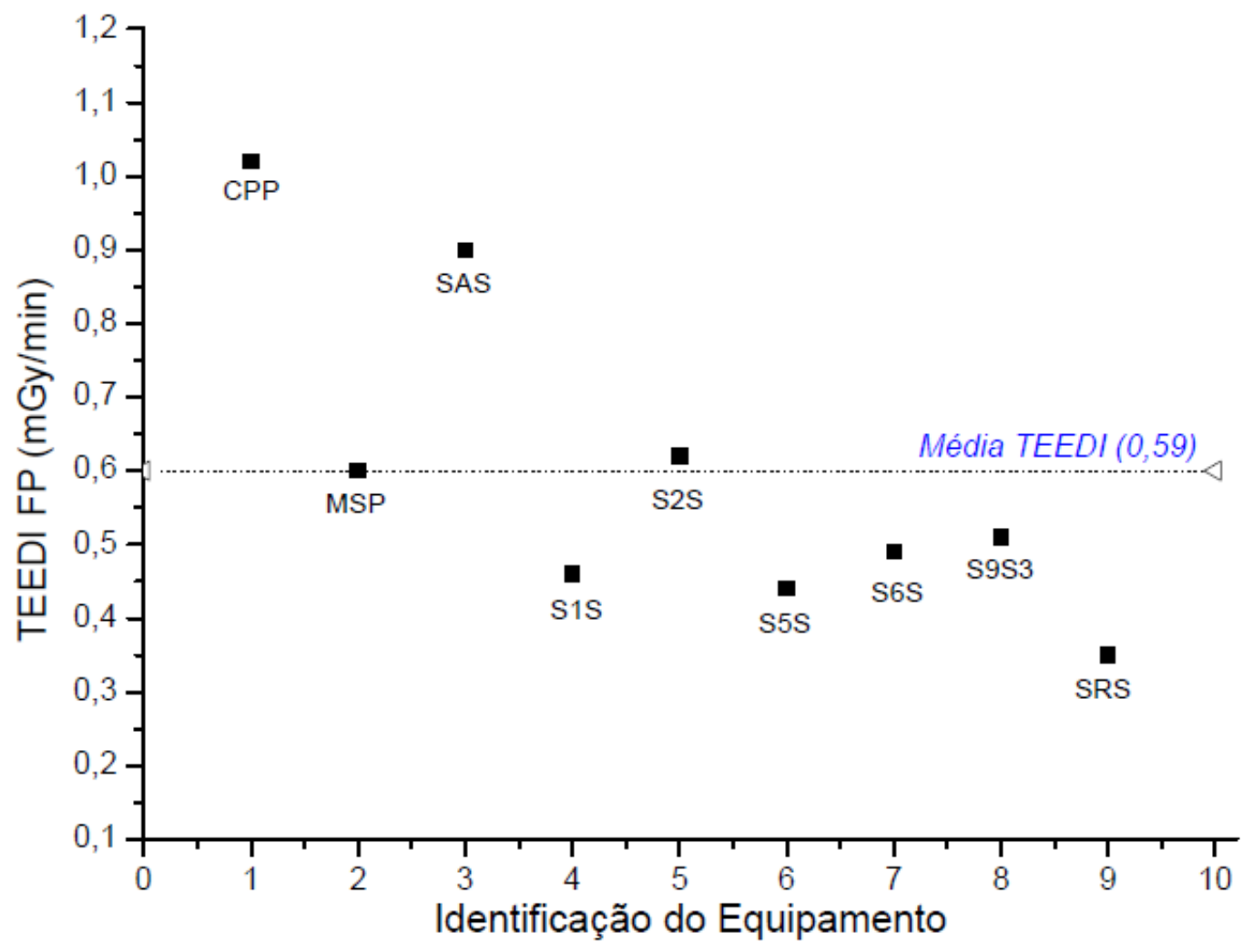

Figura 8. TEEDI FP x Identificação do Equipamento com seus respectivos códigos e o valor médio dos valores encontrados na distribuição.

A distribuição de fig 8 tem os equipamentos 1 e 3 muito acima da média, o 2 na média e o 5 praticamente nela, os 4, 6, 7 e 8 em torno de 47,5 $\mathrm{mGy} / \mathrm{min}$ e o 9 como valor mais baixo da distribuição para o FP, porém este ainda mais alto que o mais baixo para o II. O valor médio da TEEDI é maior para FP do que para II e isto vai contra o valor esperado para FP, uma vez que por ser fabricado com tecnologia mais recente deveria apresentar redução na exposição (Trianni et al, 2006). Deve-se lembrar que embora a redução no valor da exposição seja desejável, porque está diretamente relacionada à dose absorvida que o paciente incorporará, esta também tem seu comprometimento na qualidade da imagem, uma vez que doses muito baixas aumentam o ruído na imagem, podendo comprometer o diagnóstico futuro. Desta maneira, taxas de exposição muito baixas comprometem a imagem e muito altas podem comprometer a saúde do paciente e 
comprometem o princípio ALARA (ICRP, 1991) o qual preconiza que a dose absorvida deve ser tão baixa quanto razoavelmente exequível.

\section{FOM x Taxa de exposição}

As Figuras 9 e 10 apresentam resultados de FOM versus Taxa de Exposição para os equipamentos selecionados no estudo, respectivamente para os detectores II e FP.

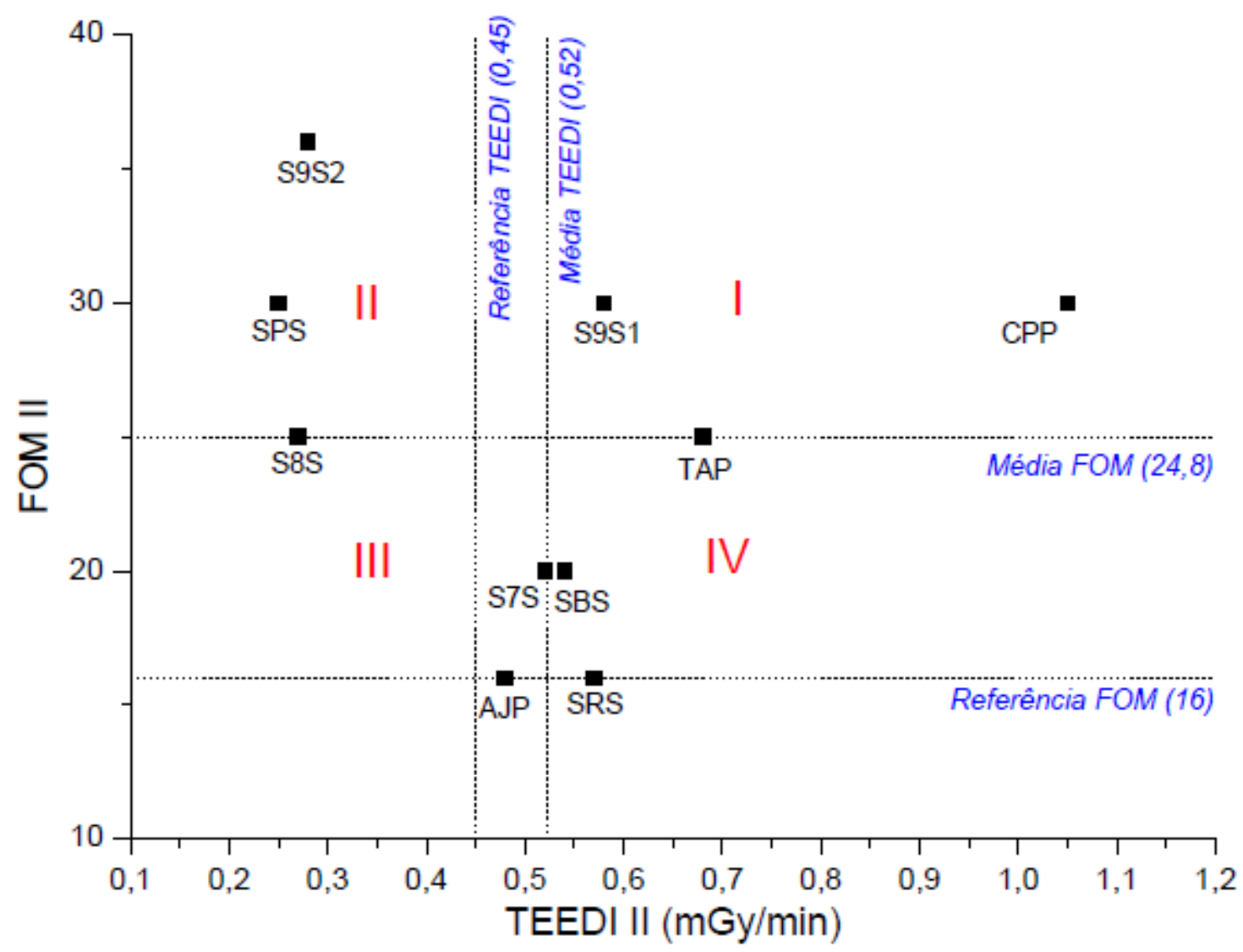

Figura 9. Resultados das medições de FOM em função das medições de TEEDI para o detector II. Os pontos representam os valores medidos para cada equipamento e as retas horizontais são os valores médios e de referência para o FOM. As retas verticais são respectivamente os valores médio e de referência para a TEEDI. 


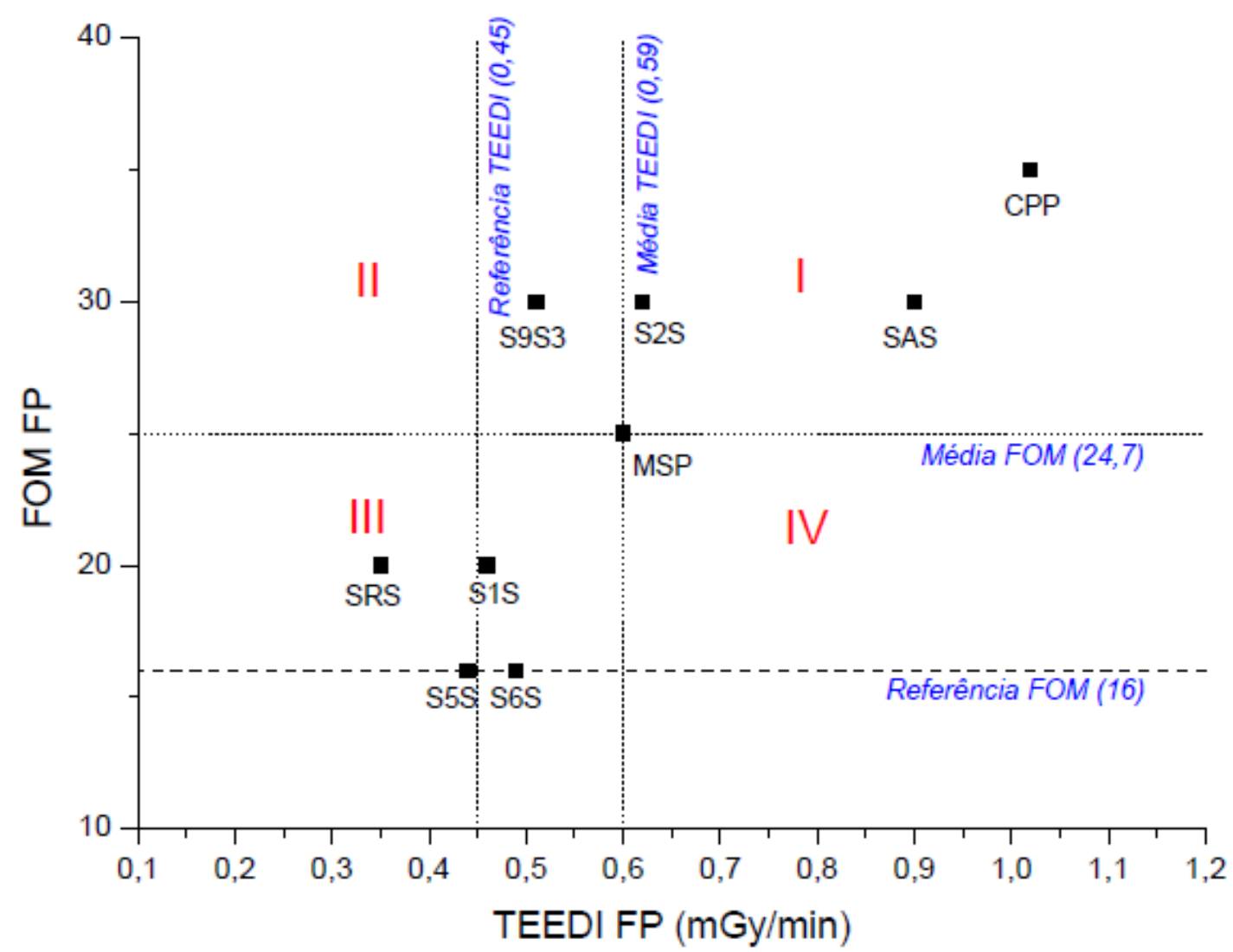

Figura 10. Resultados das medições de FOM em função das medições de TEEDI para o detector FP. Os pontos representam os valores medidos para cada equipamento e as retas horizontais são os valores médios e de referência para o FOM. As retas verticais são respectivamente os valores médio e de referência para a TEEDI.

Na Figura 9 pode-se averiguar que, das duas medidas realizadas, suas médias foram calculadas e colocadas na figura como retas tracejadas que acabaram por dividir o espaço cartesiano em quatro quadrantes I, II, III e IV. Os equipamentos que estão no quadrante I, são aqueles que apresentaram valores de TEEDI e de FOM acima da média. Neste quadrante estão os equipamentos que apresentaram potencial de otimização em TEEDI. Isto é provavelmente devido à tentativa, por parte do fabricante, de corrigir problemas de ruído excessivo nas imagens, com um ajuste maior da TEEDI. O aumento da TEEDI é uma prática relativamente comum pelo fabricante, pois é uma solução relativamente fácil e resolve o problema do ruído na imagem. Entretanto, caso se verifique, após o ajuste do sistema, que havia outras possibilidades de correção do citado problema, fica evidente que 
houve uma falha na aplicação do princípio ALARA (exposição tão baixa quanto realizável), pois haveria exposição do paciente a taxas desnecessariamente elevadas de radiação, elevando também as chances de ocorrência de seus efeitos deletérios. Esta prática de aumentar a TEEDI para minimizar o ruído na imagem, traz ainda outras consequências: a) a redução da longevidade do tubo de raios $\mathrm{X}$ e b) maiores riscos de custos médico-legais por queixas de pacientes com eritemas e/ou úlceras radioinduzidas, após procedimento de cardiologia intervencionista.

Os equipamentos que estão no quadrante II, são aqueles que apresentaram valores de TEEDI abaixo da média e FOM acima da média. Neste quadrante estão os equipamentos que apresentaram pequeno potencial de otimização em TEEDI, sendo portanto os resultados mais desejáveis do grupo. Isto é devido a ajustes cuidadosos de FOM (detalhe e contraste) e de TEEDI por parte do fabricante. O fato dos resultados estarem melhores do que os do restante da amostra, não significa que está tudo perfeito, pois ainda pode haver algum potencial de otimização. Os resultados podem ser devido à melhor qualidade dos componentes (elétricos e eletrônicos) de geração mais recente e portanto pode haver ainda alguma possibilidade da redução da taxa de exposição.

A diminuição do FOM em função da TEEDI ocorre quando esta é tão baixa que o FOM sofre queda causada pelo aumento do ruído. À medida que a TEEDI aumenta, a influência do ruído é menor e o FOM melhora, até o ponto em que outros fatores (ligados ao contraste e detalhe e eletrônica e desgastes de componente e do tubo etc...) passam a limitar o FOM e então, a cada aumento da TEEDI não ocorre mais o aumento proporcional do FOM, que no final tende a se estabilizar lentamente. Além dos pontos do quadrante I, um aumento da TEEDI poderá até causar redução do FOM, por tratar-se de uma região de saturação do detector (De Almeida et al, 1990). Nesta linha de raciocínio, esses equipamentos poderiam estar funcionando numa faixa de TEEDI em que os aumentos não trariam grandes melhorias à qualidade da imagem. Uma outra possibilidade para explicar a falta de proporcionalidade verificada nesta discussão, seria a presença de outras fontes de ruído a serem pensadas (desgaste dos componentes do gerador, corrente inerente do circuito que poderiam aumentar o ruído natural do feixe (quântico), esperado na imagem (De Almeida et al, 1990). Sendo assim, os fabricantes deveriam estar atentos para verificar este tipo de possibilidade, testando os componentes que poderiam causar esse tipo de ruído e as medições de FOM e TEEDI deveriam ser repetidas. 
Os equipamentos que estão no quadrante III são aqueles que apresentaram valores de TEEDI e de FOM abaixo das médias. Neste quadrante estão os equipamentos que apresentaram potencial de otimização em FOM. O fato da TEEDI estar abaixo da média pode estar ligado a falhas de ajustes ou de TEEDI e/ou de contraste e detalhe. Como resultado apresenta imagens com ruído significativo, causado pela falta do número de fótons suficientes para se ter uma imagem adequada. Há possibilidade também da TEEDI estar ajustada de forma adequada e haver algum problema na cadeia de imagem, tais como defeitos no detector ou o surgimento de dead pixels, que esteja prejudicando o FOM.

Os equipamentos que estão no quadrante IV, são aqueles que apresentaram valores de TEEDI acima da média e FOM abaixo da média. Neste quadrante estão os equipamentos que, de toda a amostra dos detectores II, apresentaram o maior potencial de otimização em TEEDI e em FOM, sendo portanto os resultados mais inadequados do grupo. Para esses equipamentos, será necessário também buscar possíveis ajustes na cadeia de imagem.

\section{Valores de Referência (FOM e TEEDI)}

Na Figuras 9 e 10 pode-se observar que há uma reta horizontal na altura do valor 16 para o FOM e uma reta vertical, correspondendo ao valor 45 da TEEDI para os dois detectores (II e FP). Essas retas representam os valores de referência sugeridos nos trabalhos de Cox et al, (1990), Boone et al (1993) e Shepard e Lin, (2002) que foram inicialmente colocadas na figura para serem utilizadas para comparar como o comportamento grupal dos equipamentos (média do FOM e média da TEEDI) ou como o comportamento de um determinado equipamento está em relação a essas referências; o que serviria para evidenciar os equipamentos que necessitam de uma maior atenção por parte dos fabricantes, em termos de manutenção. Pode-se notar que a média das TEEDIs para os dois detectores (II 52,2 e FP 59,9) estão relativamente próximas ao valor da referência $(45,0)$ correspondendo respectivamente a $+16 \%$ e $+33 \%$, entretanto os equipamentos com a menor e maior TEEDI para o detector II, corresponderiam à referência com $-44 \%$ e $+133 \%$, respectivamente. Os equipamentos com a menor e maior TEEDI para o detector $\mathrm{FP}$, corresponderiam à referência com $-22 \%$ e $+127 \%$, respectivamente. A média dos 
FOM para os dois detectores (II 24,8 e FP 24,7 ) distam do valor referência de $\sim 55 \%$ e os equipamentos com o menor e maior FOM para o detector II, corresponderiam à referência com $0 \%$ e $+125 \%$, respectivamente. Os equipamentos com a menor e maior FOM para o detector FP, corresponderiam à referência com $0 \%$ e 119\%, respectivamente.

Para o detector II, considerando a referência para FOM, há dois equipamentos com valores iguais e os restantes com valores maiores que os da referência, ou seja, todos seriam aceitos quanto ao citado parâmetro, mas considerando a média obtida, quatro equipamentos deveriam ser optimizados. Considerando a referência para TEEDI, três equipamentos deveriam ser optimizados para aumentar seus valores e praticamente um deveria diminuir seu valor.

Para o detector FP, considerando a referência para FOM, também há dois equipamentos com valores iguais e os restantes com valores maiores que os da referência, ou seja, também todos seriam aceitos quanto ao citado parâmetro, mas considerando a média obtida, quatro equipamentos deveriam ser optimizados. Considerando a referência para TEEDI, dois equipamentos deveriam ser optimizados para diminuir seus valores (Tsapaki et al, 2004). 


\section{Capítulo5}

\section{Conclusões}

Neste trabalho foi realizado um estudo da qualidade da imagem e taxa de exposição que chega nos detectores dos equipamentos clínicos selecionados de fluoroscopia, com detectores Intensificador de Imagem e Flat Panel. Esses resultados são importantes porque sendo o equipamento um fornecedor das imagens cardíacas que serão analisadas pelos cardiologistas para seus diagnósticos, então esse equipamento deve estar ajustado quanto à qualidade da imagem (contraste e detalhe) e à taxa de dose fornecida que está diretamente ligada à dose de radiação que será absorvida pelo paciente. Os resultados finais obtidos, através do parâmetro FOM (proveniente do contraste e do detalhe) e da taxa de exposição TEEDI, permitiram através das suas médias, situar os equipamentos em quatro áreas (identificadas por quadrantes I, II, III e IV) de potencial de otimização de qualidade de imagem e de taxa de exposição. Baixa TEEDI e alto FOM é o ideal esperado para um equipamento (quadrante II), embora que ainda possa permitir alguns ajustes. Todos os outros quadrantes merecem atenção quanto à possibilidade ou potencial de otimização em um dos dois parâmetros citados e às vezes em ambos, por exemplo: quadrante I otimização em TEEDI, III em FOM e IV em ambos, sendo que este último necessitaria de mais ajustes. A partir do protocolo e do objeto simulador desenvolvidos, respectivamente para seleção dos equipamentos e para se obter as imagens para inferir o contraste e o detalhe, as médias dos resultados obtidos para os detectores II e FP considerando: a) o FOM, pode-se inferir que em termos de qualidade da imagem eles se equivalem, respectivamente 24,8 e 24,7 e b) para a TEEDI existe uma diferença $(12,8 \%)$ entre os valores respectivos de 59,9 e $52,2 \mathrm{mGy} / \mathrm{min}$. As médias obtidas para os dois detectores II e FP quando comparadas às respostas esperadas segundo a literatura (referências) para o FOM e para a TEEDI, apresentaram diferenças de 35,5\% e 13,8\% respectivamente para II e $35,2 \%$ e $24,9 \%$ respectivamente para FP; indicando que tanto o FOM para II quanto para FP são equivalentes quando comparados com o FOM esperado (referência) e que as médias das TEEDIs de II e de FP, embora relativamente próximas $(12,8 \%)$, a de FP é praticamente o dobro daquela de II com relação àquelas esperadas. Com 
a utilização desse processo que inclui o protocolo, o objeto simulador, as avaliações do parâmetro FOM e da TEEDI e ainda as referências da taxa de exposição e FOM esperados, entre outras coisas, foi possível diagnosticar os ajustes a serem feitos nos equipamentos (na qualidade ou na taxa de exposição) que seriam comunicados aos responsáveis pela manutenção. 


\section{Referências}

Anderson JA, Wang J, Clarke GD. Choice of Phantom Material and Test Protocols to

Determine Radiation Exposure Rates for Fluoroscopy. Scient. Exhibit. 2000 JulAug;20(4):1033-1042.

Baba R, Konno Y, Ueda K, Ikeda S. Comparison offlat-panel detector and imageintensifier detector for cone-beam CT. Comput. Med. Imag. Graph. 2002 Jan;26:153-158.

Balter S, Murray K, Seibert T, Young D, Strauss K, Mitchell S. Vendor question and answer panel. Pediatr. Radiol. 2006;36(2Suppl):237-239. doi: 10.1007/s00247-006-0214-2.

Balter S. Methods for measuring fluoroscopic skin dose. Pediatr.Radiol. 2006;34(2 Suppl):136-140. doi: 10.1007/s00247-006-0193-3.

Bath M. Evaluating Imaging Systems: Practical Applications. Radiat.Prot.Dosim. 2010;139(1):26-36. doi:10.1093/rpd/ncq007.

Boone JM, Pfeiffer DE, Strauss KJ, Rossi RP, Lin PJP, Shepard JS, et al. A survey of fluoroscopic exposure rates: AAPM Task Group No. 11 Report. Med.Phys. 1993;20(3):789-794.

Bromley JF. The Eye and Radiology. Br.J.Radiol. 1994 Mar;XVII(195):65-66.

Burgess AE. The Rose model, revisited. Opt.Soc.Am.1999 Mar;16(3):633-646. 
Bushong SC. Radiologic science for technologists. 7th ed. St Louis, MO: Mosby; 2001

Canevaro L. Aspectos físicos e técnicos da Radiologia Intervencionista. Rev.Bras.Fis.Med. 2009 Out;3(1):101-115.

Cohen G, McDaniel DL, Wagner LK. Analysis of variations in contrast-detail experiments. Med.Phys. 1984 Jul/Aug;11(4):469-473.

Colbeth RE, Allen MJ, Day DJ, Gilblom DL, Harris RA, Job ID, et al. Flat-panel imaging system for fluoroscopy applications. Proc SPIE 1998;3336:376-387. doi:10.1117/12.317037.

Cowen AR, Haywood JM, Workman A, Clarke OF. A set of X-ray test objects for image quality control in digital subtraction fluorography. I: design considerations. Brit.J.Radiol. 1987 Oct;60:1001-1009.

Cox GG, Cook LT, McMillan JH, Rosenthal SJ, Dwyer SJ. Chest radiography: comparison of highresolution digital displays with conventional and digital film. Radiology. 1990 Sep;176:771-776.

Davies GA, Cowen AR, Kengyelics SM, Moore J, Sivanabthan MU. Do flat detector cardiac X-ray systems convey advantages over image-intensifier-based systems? Study comparing X-ray dose and image quality. Eur.Radiol. 2007;17:1787-1794. doi: 10.1007/s00330-006-0458-0.

De Almeida A, Ghilardi Netto T., Zimmerman R.L. Quantum Noise In Dual Energy Image. Phys.Med. 1990;6(3):243-245. 
den Boer A, Feyter PJ, Hummel WA, Keane D, Roelandt JR. Reduction of Radiation Exposure While Maintaining High-Quality Fluoroscopic Images During Interventional Cardiology Using Novel X-ray Tube Technology With Extra Beam Filtering. Circulation. 1994;89:2710-2714.

Faulkner K, Marshall NW, Lecomber AR, Kotre CJ. Establishment of Reference Doses for Examinations Using Digital Fluoroscopy. Radiat.Prot.Dosim. 1998;80(1-3):129-134.

Gagne RM, Gallas BD, Myers KJ. Toward objective and quantitative evaluation of imaging systems using images of phantoms. Med.Phys. 2006 Jan;33(1):83096.

Geise RA, O'Dea J. Radiation dose in interventional fluoroscopic procedures. Appl.Radiat.Isot. 1999;50:173-184.

Geise RA. The AAPM/RSNA Physics Tutorial for Residents. Fluoroscopy: Recording of Fluoroscopic Images and Automatic Exposure Control. Radiographics. 2001;21:227-236.

Giger ML, Doi K. Effect of pixel size on detectability of low-contrast signals in digital radiography. J.Opt.Soc.Am. 1987 May;4(5):966-975.

ICRP, 1991. 1990 Recommendations of the International Commission on Radiological Protection. ICRP Publication 60. Ann. ICRP 21 (1-3).

ICRP, 2012. Radiological protection in cardiology. ICRP Publication 120. Ann. ICRP 42(1). 
Ishibashi T, Imada N, Yamashita Y, Asou H, Matsumoto Y, Inadam S, et al. The Comparison between Dose Rates at the Interventional Reference Point of the Angiography Systems in Many Facilities. Clin.Tech. 2011 Mar;68(3):260-268.

Jiang Y, Wilson DL. Optimization of detector pixel size for stent visualization in x-ray fluoroscopy. Med.Phys. 2006;33(3):668-678. doi: 10.1118/1.2169907.

Keithley Instruments Inc. Instruction Manual. Model 35050 Dosimeter and Model 96020 Ion Chamber. 1975, Cleveland (OH).

Krain LS. Some Thoughts about the Importance of X-ray Exposure Histories for Patients. Med.Hypoth. 1992;37:225-231.

Lemoigne Y, Caner A. Archamps. Radiation Protection in Medical Physics. (FR):Springer; 2009. 192 p. (NATO Science for Peace and Security Series).

Lin PJP, Rauch P. Functionality and Operation of Fluoroscopic Automatic Brightness Control/Automatic Dose Rate Control Logic in Modern Cardiovascular and Interventional Angiography Systems. College Park, (MD): American Association of Physicists in Medicine by Medical Physics Publishing (US); 2012 Jun. 43 p. AAPM Report No 125. Report of AAPM Task Group 125.

Maolinbay M, El-Mohri Y, Antonuk LE, Jee KW. Additive noise properties of active matrix flat-panel imagers. Med.Phys. 2000 Aug;27(8):1841-1854.

Marshall NW, Faulkner K, Kotre CJ, Robson K. Analysis of variations in contrast-detail measurements performed on image intensifier-television systems. Phys.Med.Biol. 1992;37(12):2297-2302. 
Marshall NW. The practical application of signal detection theory to image quality assessment in X-ray image intensifier-TV fluoroscopy. Phys.Med.Biol. 2001 Feb;46:16311649.

Marshall W, A Mackenzie A, Honey ID. Quality control measurements for digital x-ray detectors. Phys.Med.Biol. 2010;56:979-999. doi:10.1088/0031-9155/56/4/007.

Miller DL, Hilohi CM, Spelic DC. Patient radiation doses in interventional cardiology in the U.S.: Advisory data sets and possible initial values for U.S. reference levels. Med.Phys. 2012 Oct;39(10):6276-6286.

MS/ANVISA. Ministério da Saúde. Agência Nacional de Vigilância Sanitária. Radiodiagnóstico Médico: Desempenho de Equipamentos e Segurança / Ministério da Saúde, Agência Nacional de Vigilância Sanitária. - Brasília: Ministério da Saúde, 2005. 104 p. (Série A. Normas e Manuais Técnicos).

MS/ANVISA. Ministério da Saúde. Agência Nacional de Vigilância Sanitária. - Brasília: Ministério da Saúde, Portaria MS/SVS n 453, de $1^{\circ}$ de junho de 1998. Diretrizes básicas de proteção radiológica em radiodiagnóstico médico e odontológico. Diário Oficial da União, 2/6/1998.

Nickoloff EL, Strauss KJ. Cardiac Catheterization Equipment Performance. Madison, (WI): American Association of Physicists in Medicine by Medical Physics Publishing (US); 2001 Jan. 73 p. AAPM No 70 Report. Report of Task Group 17.

Nickoloff EL. AAPM/RSNA Physics Tutorial for Residents: Physics of Flat-Panel Fluoroscopy Systems. Survey of Modern Fluoroscopy Imaging: Flat-Panel Detectors versus Image Intensifiers and More. Radiographics. 2011;31:591-602 
Seibert JA, Nalcioglu O, Roech WW. Characterization of the veiling glare in X-ray image intensified fluoroscopy. Med.Phys. 1984 Mar/Apr;11(2):172-179.

Shepard JS, Lin PJP.Quality Control in Diagnostic Radiology. Madison, (WI): American Association of Physicists in Medicine by Medical Physics Publishing (US); 2002 July. 73 p. AAPM No 74 Report. Report of Task Group 12.

Simon R, Vano E, Prieto C, Fernandez JM, Ordiales JM, Martinez D. Criteria to Optimise a Dynamic Flat Detector System Used for Interventional Radiology. Radiat.Prot. Dosim. 2008;129(1-3):261-264. doi:10.1093/rpd/ncn027.

Tapiovaara M. Image Quality Measurements in Radiology. Radiat.Prot.Dosim. 2006;117(1-3):116-119. doi:10.1093/rpd/nci707.

Thomas JA, Chakrabarti K, Kaczmarek R, Romanyukha A. Contrast-detail phantom scoring methodology. Med.Phys. 2005 Mar;32(3):807-814.

Trianni A, Bernardi G, Padovani R. Are New Technologies Always Reducing Patient Doses in Cardiac Procedures? Radiat.Prot.Dosim. 2006;117(1-3):97-101. doi: 10.1093/rpd/nci747.

Tsapaki V, Kottou S, Kollaros N, Dafnomili P, Koutelou M, Vano E, et al. Comparison of a conventional and a flat-panel digital system in interventional cardiology procedures. Brit.J.Radiol. 2004;77:562-567. doi: 10.1259/bjr/83257582. 
Vano E, Geiger B, Schreiner A, Back C, Beissel J. Dynamic flat panel detector versus image intensifier in cardiac imaging: dose and image quality. Phys.Med.Biol. 2005;50:5731-5742. doi:101088/0031-9155/50/23/022. 